Cite this: Phys. Chem. Chem. Phys., 2013

\title{
Femtisecond single-mole infrared spectroscopy of molecular clusters $\dagger$
}

15, 10702

\author{
M. A. Suhm* and F. Kollipost
}

The sensitivity limitations of Fourier transform infrared (FTIR) spectroscopy for the detection of molecular clusters formed in rarefied gas expansions can be overcome by synchronizing intense gas pulses at a low duty cycle with rapid interferometer scans. This turns the broadband FTIR approach into a universal cluster spectroscopy tool applicable from the far $\left(200 \mathrm{~cm}^{-1}\right)$ to the near $\left(8000 \mathrm{~cm}^{-1}\right) \mathrm{IR}$. It nicely complements more selective and more restricted laser-based techniques and it provides a gas-phase variant of the matrix-isolation method, the main drawback being substance consumption. A survey over the capabilities, limitations and perspectives of this high-throughput nozzle approach to cluster FTIR spectroscopy is given.

\section{Introduction}

The structure and motion of molecular clusters are revealed in much depth by vibrational spectroscopy. ${ }^{1}$ In contrast to microwave techniques, which can provide detailed structural and

Institut für Physikalische Chemie, Universität Göttingen, Tammannstr. 6, D-37077 Göttingen, Germany.E-mail:msuhm@gwdg.de

$\dagger$ This work is dedicated to M. Quack on the occasion of his 65th birthday. some dynamical information, ${ }^{2}$ no permanent dipole moment is required. The production and detection limits of incoherent infrared radiation have traditionally called for laser based techniques. ${ }^{3}$ The synthetic workhorse in the field of molecular clusters is a supersonic jet expansion from a dense gas reservoir into a vacuum chamber, ${ }^{4}$ during which adiabatic cooling converts internal energy into concerted translation and allows intermolecular forces to lock the molecules with each other. Optically thin seeded supersonic jet expansions required for

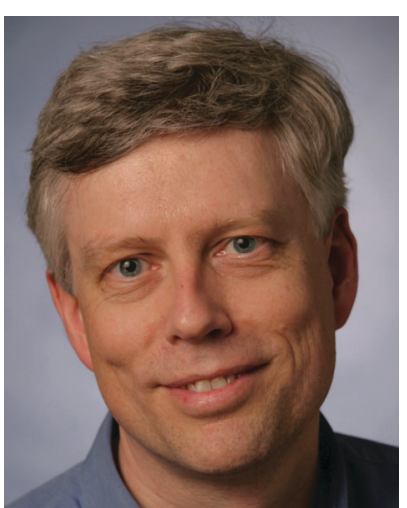

M. A. Suhm
Martin Suhm completed a Chemistry degree at the University of Karlsruhe (1985) and a PhD at the ETH Zürich (1990). Pre- and post-doctoral stays involved Canberra and Boulder/CO, the habilitation was obtained in Zürich (1995). Since 1997, he has been a professor of Physical Chemistry at the University of Göttingen. He served the Editorial board of PCCP from 2006 to 2011. His research focusses on hydrogen bonds and other intermolecular interactions, as probed by vibrational spectroscopy. With 1-6 papers in each volume of PCCP so far, he has been trying to advocate the power and versatility of linear infrared and Raman spectroscopy in elucidating intermolecular dynamics and in benchmarking quantum chemistry tools. This Perspective builds on his very first PCCP paper in volume 1 (1999).

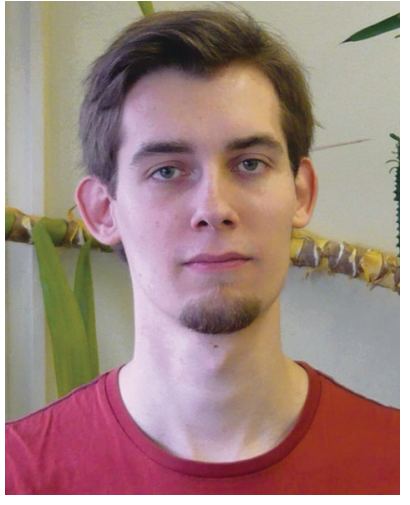

F. Kollipost
Franz Kollipost got his Chemistry diploma at the University of Göttingen in 2009 and is currently completing his PhD. $\mathrm{He}$ is extending the large slit-jet FTIR spectrometer in the Göttingen laboratory further into the challenging far- and nearinfrared regions, besides exploring its power in the midinfrared. His research interest focusses on the vibrational dynamics of hydrogen bonded clusters. He is co-supervised by René Wugt Larsen from DTU. 
cold cluster generation and the wish for conformational and size-selectivity have sometimes led to a preference of action spectroscopy ${ }^{5}$ over direct absorption techniques, although the latter may offer advantages in terms of the connection to theoretical predictions of spectral intensities. ${ }^{6}$ In spite of the overwhelming success of laser spectroscopy in particular in the high resolution ${ }^{7}$ and double resonance ${ }^{8}$ regime, demand for panoramic tools like FTIR spectroscopy which provide simultaneous access to different spectral regions, including the difficult far- and near-infrared ranges, has remained high. ${ }^{9}$ The ability to record a complete and unbiased picture of the supersonic jet expansion and of the cluster dynamics remains attractive and has provided an important niche for the interferometric FTIR approach using incoherent light sources. ${ }^{10}$

The combination of FTIR spectroscopy with supersonic jets has been reviewed expertly and exhaustively in $2000,{ }^{11}$ two decades after its first realization. ${ }^{12}$ Before 2000 , it was predominantly realized with continuous expansions, whereas the present work advocates pulsed variants which are particularly useful for molecular clusters and other low- to moderateresolution applications. These pulsed jet variants were started less than two decades ago at a small scale in the Quack group. ${ }^{13-15}$ They were scaled up and broadened in scope since 1998 in Göttingen. ${ }^{10,16,17}$ A key to the successful scale-up is a relatively low duty cycle, which leads to waiting times between pulses on the order of 50 seconds, as detailed below. This is encoded in the first word of the Perspective title, as femti means fifty in Norwegian.

The principles and perspectives of this pulsed supersonic jet-FTIR approach for the characterization of structural, dynamical and energy aspects for molecular clusters shall be outlined in the following, setting it into the context of other approaches with related goals. Some emphasis will be put on the 'difficult' spectral ranges above and below the mid-infrared, after presenting the success for specific fundamental vibrations with high sensitivity to intermolecular interactions.

\section{The concept of high throughput pulsed jet-FTIR spectroscopy}

FTIR absorption spectroscopy records the interferogram of a broadband light source (typically a tungsten filament or a glowing $\mathrm{SiC}$ rod) as a function of optical path difference between the two interferometer arms, either continuously or step-by-step, in the presence and absence of an inserted sample. The longer the path difference, the higher the achievable resolution. The finer the sampling intervals, the larger the spectral range covered simultaneously. Multiple fast scans contribute to noise reduction, short times between these sample and reference measurements contribute to the stability of the spectral baseline. In a supersonic jet expansion through a pinhole $\mathrm{e}^{12,18}$ or slit nozzle ${ }^{19}$ into a vacuum chamber, the switch between the sample and the reference can be carried out in a short time by opening and closing the nozzle or the gas supply using a valve. ${ }^{10}$

\subsection{Pulsed operation over entire scans}

For a given vacuum pump capacity, pulsed supersonic jets offer a much higher molecular density than continuous jets, ${ }^{20-22}$ if the system tolerates pressure jumps. This allows for dilution of the compound under investigation in an excess of a carrier gas, typically $\mathrm{He}$ or Ar, with benefits for cooling and controlled aggregation. Such seeding in gases with no or few internal degrees of freedom rules out traditional forms of cryopumping. ${ }^{12,23,24}$ Intense pulses further rule out diffusion pumps and turbomolecular pumps, as we will see. This leaves rugged mechanical pumps as the preferred option for vacuum recovery. The natural realization of pulsed operation might thus appear to consist in step-scanning (or even rapid-scanning) the interferometer mirror, pulse by pulse. Suitable short gas pulses are technically feasible. ${ }^{25}$ Despite its potential for time-resolved measurements, this solution has not yet been applied routinely, ${ }^{26,27}$ partly because the step-scan mode is more prone to low frequency noise and drifts than the rapid continuous scan mode which standard spectrometers offer. Asynchronous pulsing is an option to exploit the higher peak clustering in intense pulsed nozzles while maintaining the advantages of rapid continuous scanning, ${ }^{13}$ but it was quickly replaced by a synchronized variant. ${ }^{13}$

Indeed, it makes sense to scan the interferometer as quickly as possible, and to extend the gas pulse over the entire scan. This is not practical for long high-resolution scans, unless one decomposes the interferogram into several sections. ${ }^{16} \mathrm{How}^{-}$ ever, it becomes feasible if the scan lasts for less than about a second, i.e. in the low (1-5 $\left.\mathrm{cm}^{-1}\right)$ resolution mode which is useful for most molecular cluster spectra. By synchronising the gas pulses with such short interferometer scans, one can record the reference spectrum immediately before (or soon after) the gas pulse, with advantages for baseline stability. Because the entire spectral range is recorded simultaneously, even an unstable cluster source yields a faithful spectrum.

\subsection{Low duty cycle as an advantage}

One has to allow for a recovery period after the intense gas pulse, before the next pulse can be probed. It is advantageous to work in a regime where the gas flow by far exceeds the pumping capacity of the system. Otherwise, there would be no molecular density advantage over continuous operation. In order to keep the shock waves of the expansion outside the optical sampling region, ${ }^{10}$ the residual background gas level has to be sufficiently low. For typical stagnation pressures on the order of 1 bar, nozzle diameters of $0.2 \mathrm{~mm}$ and sampling areas on the order of $1 \mathrm{~cm}^{2}$, this means that the background pressure should not exceed $0.1 \mathrm{mbar}$ in a pinhole expansion or $1 \mathrm{mbar}$ in a long slit expansion. ${ }^{28}$ The difference already shows that a slit geometry is favorable, besides its larger propensity for clusters $^{19}$ due to a slow decay of the molecular density with nozzle distance. The resulting large number of molecule-cluster collisions can promote isomerizations and bring the aggregates closer to thermodynamic equilibrium. ${ }^{29}$ Moreover, the crosssection of a slit can be easily scaled up by extending its length, 
as long as the infrared beam can sample the full length. Cross sections of $120 \mathrm{~mm}^{2}$ are technically achievable (corresponding to a slit length of $600 \mathrm{~mm},{ }^{17}$ see Fig. 2), if the pulsed valve fills a preexpansion chamber instead of sealing the entire slit. However, this implies gas flows on the order of 10 standard- $\mathrm{dm}^{3}$ per pulse of $1 \mathrm{~s}$ duration, which require a buffering vacuum chamber of about $20 \mathrm{~m}^{3}$ size to be diluted down to a residual pressure on the order of 0.5 mbar. The post-pulse background pressure (typically 0.7 mbar) is then pumped down to about 0.2 mbar in a time period of about $50 \mathrm{~s}$ ('femti' seconds in Norwegian and intraScandinavian communication, hence femtisecond spectroscopy, see Fig. 1) at a pumping speed of $2000-2500 \mathrm{~m}^{3} \mathrm{~h}^{-1}$, before the next gas pulse increases it again by $0.5 \mathrm{mbar}$. This translates into a very low duty cycle on the order of $2 \%$.

It is rewarding to compare these numbers to a hypothetical continuous operation mode, using the same average pumping speed. The nozzle (or stagnation pressure) would have to be $50 \times$ smaller, hence also the peak absorbance of a simple system. To achieve the same signal-to-noise ratio, the scan time would have to amount to $2500 \mathrm{~s}$, instead of $1 \mathrm{~s}+50 \mathrm{~s}$ waiting time. Therefore, a 49-fold time (and compound) saving is predicted for the pulsed operation. Alternatively, in the same amount of time, a $7 \times$ better signal-to-noise ratio is achieved by the pulsed operation. Furthermore, the idle time can be used to record essentially noise-free and still almost instantaneous multiple background scans (Fig. 1), which would have to be added later in a continuous operation. To achieve the same performance by increasing the pumping speed, pumps with a speed of $125000 \mathrm{~m}^{3} \mathrm{~h}^{-1}$ would have to be employed. It is obviously much more economical to invest into buffer vacuum chambers than into vacuum pumps, as long as the flow through the nozzle can be scaled up accordingly. The current limit of $600 \mathrm{~mm}$ slit nozzle length is largely determined by the need of keeping the IR beam waist sufficiently narrow over the full distance (Fig. 2).

In the case of low speed detectors such as He-cooled bolometers, the pulse length can be extended. In no case, more than about $1 \mathrm{~mol}$ of carrier gas flows through the supersonic nozzle during a single pulse. This already indicates a major weakness of the technique - its high carrier gas consumption. The compound of interest itself is typically present at a dilution of $0.1-1 \%$. Considering that 100-1000 coadded scans (and sizeable quantities of carrier gas) are needed to detect the weakest signals, the molecular substance consumption for a high quality spectrum also approaches $1 \mathrm{~mol}$ (hence: singlemole spectroscopy). However, in the case of rare isotopes, useful spectra have been obtained with as little as $300 \mathrm{mg}$ of sample. ${ }^{30}$ Further details on this (tongue in cheek) 'femtisecond single-mole' infrared cluster spectroscopy approach based on high-throughput pulsed nozzles may be found in a range of publications, summarized in the next section. An interesting concept to reduce gas consumption is the introduction of a closed-cycle compressor system. ${ }^{31}$

\subsection{A menu of nozzles}

The numbers discussed in the previous section apply to the $600 \times 0.2 \mathrm{~mm}^{2}$ slit nozzle spectrometer connected to $23 \mathrm{~m}^{3}$ of

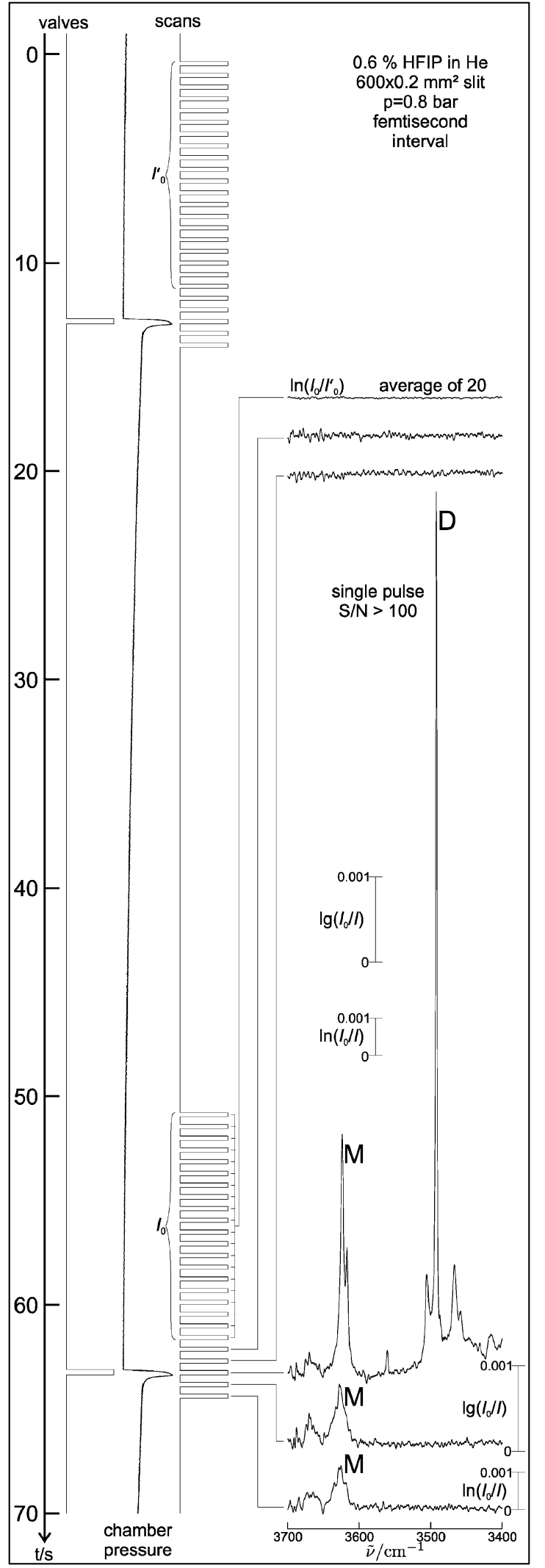

Fig. 1 Time trace of a typical pulsed jet-FTIR experiment over $70 \mathrm{~s}$, showing a valve opening at $13 \mathrm{~s}$ (and $63 \mathrm{~s}$ ) and the resulting chamber pressure, 20 background scans, 2 pre-pulse scans, 1 probe scan and 2 post-pulse scans, as well as typical spectra of HFIP (see Section 7 and Fig. 5) in the $\mathrm{OH}$ stretching range with a single pulse $\mathrm{S} / \mathrm{N}$-ratio in excess of 100 resulting from these scans. 


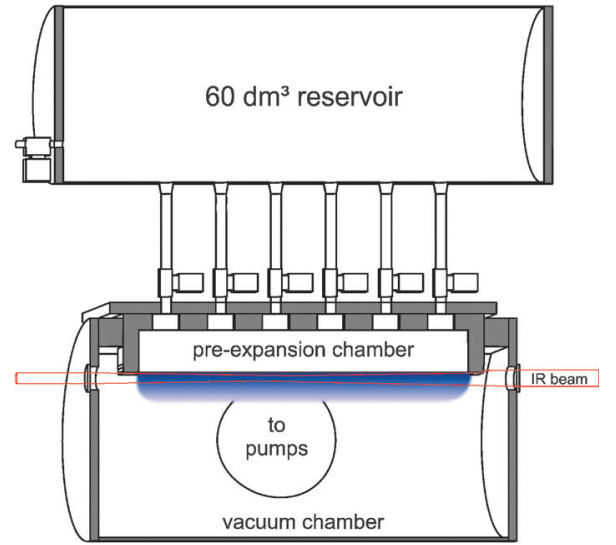

Fig. 2 Schematics of the adjustable $600 \mathrm{~mm} \times 0.2 \mathrm{~mm}$ slit nozzle ${ }^{17}$ with a gas reservoir, 6 feeding magnetic valves, a pre-expansion chamber, the expansion into the cylindrical vacuum chamber and the FTIR beam.

vacuum buffer and pumped at $2500 \mathrm{~m}^{3} \mathrm{~h}^{-1}$, which in the laboratory jargon is denoted filet-jet (fine but lengthy) due to its filet-shape expansion zone. ${ }^{17,32}$ Somewhat reduced pulsed operation advantages apply to the original $120 \times 0.3 \mathrm{~mm}^{2}$ slit nozzle with active slit sealing, connected to $10-23 \mathrm{~m}^{3}$ of vacuum buffer and pumped at $2000 \mathrm{~m}^{3} \mathrm{~h}^{-1}$ (ragout jet ${ }^{10}$ ) as well as to the heatable popcorn (poppet-controlled resistively heated ozzle $^{33}$ ) jet with two parallel $10 \times 0.5 \mathrm{~mm}^{2}$ slits, connected to $4 \mathrm{~m}^{3}$ of vacuum and pumped at $500 \mathrm{~m}^{3} \mathrm{~h}^{-1}$. These jet setups are combined with a range of rapid-scan Bruker spectrometers (IFS 66v/S, Equinox 55). All acronyms have in common an international food designation, as well as some second or even third hidden meaning. An important key to the success of all these setups is the possibility of working at relatively high background pressures on the order of 1 mbar. While this rules out diffusion and turbomolecular pumps, it allows for enormous gas throughput at moderate mechanical pumping speeds. Due to the use of slit nozzles, the zone of silence $^{4}$ remains sufficiently large for FTIR probing, although one should always try to minimize the optical path outside the expansion region to reduce background absorption. Part of the undesired warm background absorption can actually be compensated by recording the background spectrum not too long after the pulse, instead of before the pulse.

The realization of the short gas pulse deserves some discussion. In contrast to the (sub-)ms pulse time scale of typical laser jet spectrometers, our upscaled FTIR approach allows for more flexibility. The magnetic solenoid approach popular in pinhole and small slit nozzles ${ }^{21}$ is still possible, but neither very practical (because of the increasing mass of the sealing piston) nor necessary (because of the relaxed requirements on the opening and closing times). In the ragout-jet, ${ }^{16}$ solenoid valves operate on a pressurized air mechanism to lift the piston. In the filet-jet, ${ }^{32}$ a series of six high throughput solenoid valves fill a pre-expansion chamber, from which the expansion through the long slit is formed (Fig. 2). In the smaller popcorn-jet, ${ }^{33,34}$ the heated compound is enclosed between two check valves which remain closed below a threshold pressure. When the solenoid valve supplies the carrier gas pulse, the check valves transiently open and release the vapor phase above the heated sample reservoir into the carrier gas. The dead volume associated with the filet and popcorn variants is tolerable compared to the overall gas flow. It even offers the possibility of recording 'fading pulse' spectra along with the core pulse spectra of interest after the solenoids have closed (Fig. 1). These spectra usually involve much less clusters and allow for a discrimination between weak monomer and dimer or larger oligomer signals.

An entirely different regime of jet expansions is probed in the RESS process, the rapid expansion of supercritical (and subcritical) solutions. The pulsed vacuum variant realized in Göttingen ${ }^{35}$ builds on the same buffering principle as the slit jet setups and compensates the very small nozzle cross section by pressures up to 400 bar. It allows to vibrationally characterize low-volatility molecules co-expanded in the $\mathrm{CO}_{2}$ carrier. Many other pulsed and continuous expansion setups are available ${ }^{36-38}$ but they typically employ more number density sensitive detection methods.

Laval slit nozzles with their constrained flow closer to thermodynamic equilibrium are also in use for the probing of clusters by FTIR spectroscopy. ${ }^{31,39}$ However, the most powerful continuous Laval nozzle cluster experiments are axially symmetric $^{40,41}$ and have been developed for low temperature kinetic experiments. ${ }^{42}$

A useful comparison of the advantages and disadvantages of turbomolecular and Roots type pumps in jet-FTIR spectroscopy is given by Herman et $a l^{43}$ For high-resolution, very low temperature studies of molecules, the former may be advantageous, whereas the high throughput nozzles advocated here for molecular clusters are better pumped with the latter, due to their tolerance for higher background pressures.

Continuously operated slit jet FTIR setups based on mechanical pumps may be classified into two different categories. In the first type, the pumping capacity is comparable to or up to three times larger than that available in our long pulsed slit jet setup. This means that the slit cross section has to stay significantly below $10 \mathrm{~mm}^{2}$. Examples include the Texas setup ${ }^{44,45}$ some of the Brussels designs ${ }^{11,46}$ or the recent jet-AILES experiment. ${ }^{47}$ They can achieve near- or even subDoppler resolution and are often more focussed on isolated molecules. In order to study molecular clusters in continuous slit jets, it is better to have pumps which are a full order of magnitude larger, such as in the PNNL experiment ${ }^{48}$ and in the well characterized Rennes slit jets. ${ }^{49,50}$

Of course, the transition between molecules and molecular clusters is a continuous one, with some of the latter being rather strongly bound. ${ }^{51,52} \mathrm{~A}$ better distinction may refer to the vibrational partition function at room temperature. If it exceeds $10^{2}-10^{3}$, it becomes progressively more difficult to achieve sufficient vibrational cooling in the jet for rotationally resolved spectra, which would justify a large optical path difference in the interferometer.

The option of heating the valve $e^{21,47,53,54}$ is an obvious requirement to entrain low-volatility samples into the carrier gas. 
It can also be advantageous for the study of monomers and dimers of more volatile compounds, because the high temperature prevents larger cluster formation even at relatively high concentration. However, the extent of rotational and in particular vibrational cooling after the nozzle suffers. On the other hand, high energy conformations might be generated at elevated temperature and then be frozen out in the expansion, thus giving access to thermodynamically stable but kinetically hindered dimers. ${ }^{54}$

\subsection{Trade-off between resolution and radiation throughput}

Continuous and pulsed slit jet sources are also realized in laser experiments on molecular clusters, including diode lasers ${ }^{55-59}$ and difference frequency lasers. ${ }^{21}$ Their key advantage is intrinsically high resolution, where slit jets offer the extra benefit of Doppler width reduction, whereas the FTIR approach develops its full strength when a survey over a wide range is required or rotational resolution is out of reach due to the size of the system.

The price to pay for an increase in resolution is substantial in tabletop single-pass FTIR jet spectroscopy. Not only does the scan time increase in proportion with the resolution requirements, but also the noise level grows due to the increased bandwidth. For non-resolved bands, this is not compensated by an increased peak absorbance. Furthermore, the aperture of the instrument has to be reduced to avoid self-apodisation. The latter handicap is not critical for highly brilliant synchrotron light sources. ${ }^{47}$ However, for applications to hydrogen-bonded clusters with their intrinsically broader bands, a spectral resolution between $0.5 \mathrm{~cm}^{-1}$ and $5 \mathrm{~cm}^{-1}$ is usually adequate and profits from maximum light throughput, if matched by large area detectors.

Instead of having a single optical pass through a long slit nozzle expansion, one could consider multipassing the IR light through a shorter slit expansion. However, this would again require closing the aperture of the infrared source far beyond the diameter tolerated by the low resolution FTIR scan. Much of the path length gain would be lost by this intensity reduction. This is different in high resolution studies, ${ }^{47,60}$ and less of a problem for axially symmetric Laval nozzles. ${ }^{41}$

For long slit jets, instead of closing the aperture, it is advantageous to open it as far as possible and to reduce the total photon density on the detector using band pass filters and by using larger detector areas. The latter is less of an advantage in the mid-IR due to detector noise, but it offers an excellent trade-off for shot noise limited detection ${ }^{21}$ in the near IR (InSb, InGaAs), also releasing the requirements for the focussing optics. Furthermore, the preamplifier of the detector has to be adjusted to the light level in order to avoid losses in the analog-to-digital conversion. Because gain-ranging can be used in the Bruker IFS $66 \mathrm{v} / \mathrm{S}$ up to $80 \mathrm{kHz}$, this is a preferred choice for fast detectors, although higher speeds might be advantageous in the absence of gain ranging.

\subsection{Femtisecond single-mole spectroscopy for molecular clusters}

In summary, the combination of rapid-scan infrared interferometry, large spectrometer aperture, long slit expansion, single-pass absorption, single-scan coverage of the pulse, multiple pre- or post-pulse background recording, very large vacuum buffer, moderate size mechanical pumps and low duty cycle is found to be well tailored to provide automated high quality FTIR spectra of molecular clusters at moderate spectral resolution, if the molar dimensions of substance consumption can be tolerated. In the following, selected results achieved in different spectral ranges will be set into the context of related studies using other techniques.

\section{The hydride stretching range}

The stretching fundamentals localized on $\mathrm{OH}^{61}$ and $\mathrm{NH}$ groups $^{62}$ are sensitive markers for hydrogen bond formation. The same is true for the hydrogen halides, which were among the first systems whose clusters were studied in supersonic jet expansions. $^{63,64}$ The existence of hydrogen bond donor and acceptor sites in immediate neighbourhood leads to strong polarization and hence cooperativity effects. Consequently, these systems have been explored in the first high-throughput pulsed FTIR experiments. ${ }^{10,15,65}$ The pronounced cooperativity mediated via the HX bond enables spectral size resolution without size selection up to at least tetrameric aggregates. The onset of puckering in the hydrogen-bonded ring systems reflects itself in the spectra. ${ }^{66}$

Alcohols show closely related aggregation patterns ${ }^{10}$ and have been studied quite systematically, ${ }^{67,68}$ including the connection to molecular force fields. ${ }^{34}$ The dimers lend themselves to detailed comparison with theory, ${ }^{69}$ also in terms of relative hydrogen bond donor/acceptor strengths. ${ }^{30}$ The $\mathrm{OH}$ dimerization shift of the methanol dimer is anomalously small, ${ }^{10,67}$ in part due to off-diagonal anharmonicity effects caused by the small mass of the monomer units. The role of branched ring topologies is a very minor one at least up to pentameric aggregates, $^{70}$ unless the internal energy of a metastable complex is removed quickly in a helium droplet environment. ${ }^{71}$

The case of water is considerably more intricate due to the more diverse hydrogen bond topologies starting at 6 monomer units, ${ }^{61}$ but narrow absorption bands have been demonstrated in FTIR jet experiments, ${ }^{16}$ also in comparison to cavity ring down (CRD) studies. ${ }^{72}$ Recently, water cluster spectra were also obtained in a continuous jet-FTIR experiment. ${ }^{50}$ Mixed wateralcohol clusters help in elucidating relative donor and acceptor strengths and excess heats of mixing. ${ }^{73}$

Mixed water-hydrogen halide clusters serve as prototypes for the onset of electrolytic dissociation, ${ }^{74,75}$ also in related systems. ${ }^{76}$ While it has been shown that up to three waters still leave the $\mathrm{HCl}$ bond in place, ${ }^{77,78}$ the lack of size selectivity in jet-FTIR spectra prevents a statement on the effect of four water molecules. Recent claims of a zwitterionic structure by helium droplet techniques ${ }^{79}$ have been refuted by others ${ }^{80,81}$ and the relevant absorption band claimed to correspond to an earlier vaguely assigned undissociated cluster. ${ }^{77}$ It is likely that molecular and zwitterionic isomers coexist over a certain range of solvent molecules ${ }^{76}$ and further studies will be needed to obtain a complete picture in this difficult size range. The relative stability 
of such acid-solvent clusters and their cooperativity patterns can be tuned by substituting methanol for water ${ }^{82}$ and by varying the acid. ${ }^{77}$ From a vibrational coupling perspective, it is likely that the spectral signature of zwitterionic clusters is broader than that of more weakly coupled undissociated isomers, like in the case of the strong hydrogen bonds of carboxylic acid dimers. ${ }^{16}$ The latter show Fermi resonance couplings with molecular framework overtones and combinations, which are switched on by the strong red shift of the antisymmetric $\mathrm{OH}$ stretching mode. ${ }^{83}$

In $\mathrm{NH}$ hydrogen bonds formed by pyrazole, ${ }^{32}$ the onset of such Fermi resonances only occurs for trimers and larger clusters, because the hydrogen bonds in dimers are too strained to induce a sufficiently large red shift. ${ }^{84}$ Weaker interactions are found towards $\pi$-systems - a field where the performance of CRD and FTIR jet techniques may be directly compared..$^{85,86}$ The volatility of amino compounds changes dramatically with hybridization, such that amino esters can be studied without heating the nozzle, ${ }^{87,88}$ whereas molecules containing amide bonds profit from high temperature nozzles. ${ }^{54,89}$ IR intensity is also strongly dependent on the hybridization state, as well as on temperature. ${ }^{88}$ A particularly interesting case is found for pyrrolidine, ${ }^{90}$ with an almost negligible monomer $\mathrm{NH}$ stretch intensity and up to three orders of magnitude intensity gain in the hydrogen-bonded dimer mode. Another effect of hydrogen bonding in this case is to switch the energetical preference of the $\mathrm{NH}$ bond from equatorial to axial. A related dimer is that of dimethylamine, ${ }^{91}$ which has been studied at environmental temperatures. Due to thermal excitation, the reported NH complexation shift of $-35 \mathrm{~cm}^{-1}$ is likely much smaller than the $0 \mathrm{~K}$ shift. The latter is possibly closer to the axial pyrrolidine shift of $-64 \mathrm{~cm}^{-1}$, but an experimental verification is not yet available.

The routine nature of jet-FTIR spectroscopy allows for the study of hydrogen bond acceptor trends such as for pyrrole as a donor. ${ }^{86}$ Among others, benzene was used and a red-shift of $46 \mathrm{~cm}^{-1}$ was observed. A recent UV/IR double resonance study ${ }^{92}$ argues that the correct shift is $59 \mathrm{~cm}^{-1}$ and the peak observed in our FTIR work ${ }^{86}$ may be due to an impurity acceptor. However, one should not dismiss the possibility that the peak observed in UV/IR double resonance corresponds to a trimer built from one pyrrole and two benzene units, due to complex fragmentation processes upon excitation/ionization. ${ }^{93}$ Re-analysis of our FTIR data $^{86}$ tends to confirm that the pyrrole-benzene dimer has a redshift of $46 \mathrm{~cm}^{-1}$, whereas satellite peaks with $60 \mathrm{~cm}^{-1}$ and $67 \mathrm{~cm}^{-1}$ red shift are more likely due to one pyrrole with two and three benzene molecules, respectively. A joint FTIR and UV/IR double resonance study of the related pyrrole-toluene system is planned to resolve the issue. Such combined studies using linear, but non-selective FTIR spectroscopy and selective double-resonance UV/IR spectra are now also feasible for more strongly bound systems, ${ }^{94,95}$ because the vapor pressure limitation of our FTIR approach has been relaxed by employing heated nozzles.

For complexes of pyrrole with acetone, UV/IR double resonance results are not available. However, the composition of mixed dimers and trimers was derived rather unambiguously from a CRD study of mixed expansions. ${ }^{96}$ The only problem is the apparent elusiveness of the most stable $\mathrm{Py}_{2} \mathrm{Ac}$ trimer, a cyclic PyPyAc structure with $\mathrm{NH} \cdots \pi-\mathrm{NH} \cdots \mathrm{O}=\mathrm{C}$ topology closed by dispersion forces and residual polar interactions. Because only one unassigned $\mathrm{NH}$ stretching band remained in the CRD spectrum, it was tentatively assigned to a doubly

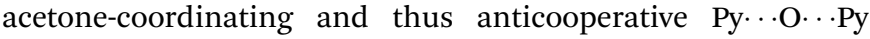
structure, which is about $20 \mathrm{~kJ} \mathrm{~mol}^{-1}$ higher in energy. ${ }^{96}$ As this appears very unlikely on energetic grounds, we decided to reinvestigate the system using jet-FTIR spectroscopy. We fully confirm the CRD spectra and concentration dependencies of the peaks (see Fig. 3, top). Therefore, the only remaining possibility is accidental band overlap of two clusters within the $3335 \mathrm{~cm}^{-1}$ band, which was assigned exclusively to the $\mathrm{PyAc}_{2}$ cluster. ${ }^{96}$ We confirm this dominant assignment of the CRD study, but accepting a second contribution by $\mathrm{Py}_{2} \mathrm{Ac}$ together with the $\mathrm{Py}_{2} \mathrm{Ac}$ band at $3406 \mathrm{~cm}^{-1}$ would reconcile the spectra with the more stable cyclic cluster. To corroborate this alternative and energetically much more plausible assignment, we have carried out two complementary experiments. First, we have repeated the experiment using fully deuterated acetone. Due to subtle anharmonic zero point energy effects, per-deuterated acetone is expected to be a slightly better $\mathrm{C}=\mathrm{O}$ hydrogen bond acceptor. ${ }^{30}$ Therefore, NH stretching vibrations connected to acetone are expected to experience a slight red-shift, which is easily detected by FTIR down to about $10 \%$ of the spectroscopic resolution of $2 \mathrm{~cm}^{-1}$ due to its intrinsic interferometer calibration. Indeed, the PyAc dimer NH-band is red-shifted by $0.7 \mathrm{~cm}^{-1}$, whereas the nominal $\mathrm{PyAc}_{2}$ trimer band is only shifted by $0.4 \mathrm{~cm}^{-1}$. More importantly, the band at $3406 \mathrm{~cm}^{-1}$ is blue-shifted by $0.3 \mathrm{~cm}^{-1}$, which is not consistent with a PyAcPy assignment but rather with the $\mathrm{NH} \cdots \pi-\mathrm{NH}$ hydrogen bond in a PyPyAc trimer. While this provides some indirect support for cyclic PyPyAc trimer contributions at 3406 and $3335 \mathrm{~cm}^{-1}$, a more robust experiment was deemed necessary. After replacing acetone by cyclopentanone (Cp), substantial shifts of the bands are expected, while preserving the general hydrogen bond topology preference of pyrrole-ketone systems. As shown in Fig. 3 (bottom part), this indeed lifts the accidental degeneracy of the two mixed trimer contributions at $3335 \mathrm{~cm}^{-1}$ and reveals the cyclic $\mathrm{Py}_{2} \mathrm{Cp}$ trimer at $3309 \mathrm{~cm}^{-1}$, now well separated from the PyCp $\mathrm{p}_{2}$ trimer at 3325 and $3329 \mathrm{~cm}^{-1}$ (split into a doublet due to ring puckering motion, isomerism or Fermi resonance). Again, the red-shift due to Ac $\rightarrow \mathrm{Cp}$ replacement is smallest for the band near $3400 \mathrm{~cm}^{-1}$, underscoring its origin in an $\mathrm{NH} \cdots \mathrm{N}$ rather than an $\mathrm{NH} \cdots \mathrm{O}$ hydrogen bond. We can thus reconcile the CRD and FTIR spectra of Py/Ac coexpansions in the presence of the by far most stable cyclic PyPyAc trimer.

While pyrrole and its derivatives are rather volatile, ${ }^{87}$ most compounds with $\mathrm{sp}^{2}$-hybridized $\mathrm{NH}$ groups and strong hydrogen bond acceptors require heated nozzles due to their stronger hydrogen bond interaction. Dimers of the four simple peptide bond models formamide, acetamide, $N$-methylformamide and $\mathrm{N}$-methylacetamide could be studied in isolation by direct absorption spectroscopy. ${ }^{33}$ The goal here is to extend the FTIR studies to UV-chromophore-free protected peptides, their folding ${ }^{62,97}$ and their $\beta$-sheet-like ${ }^{98}$ aggregation. ${ }^{99}$ Recent results for protected alanine ${ }^{89}$ indicate that the $\beta$-sheet aggregation 


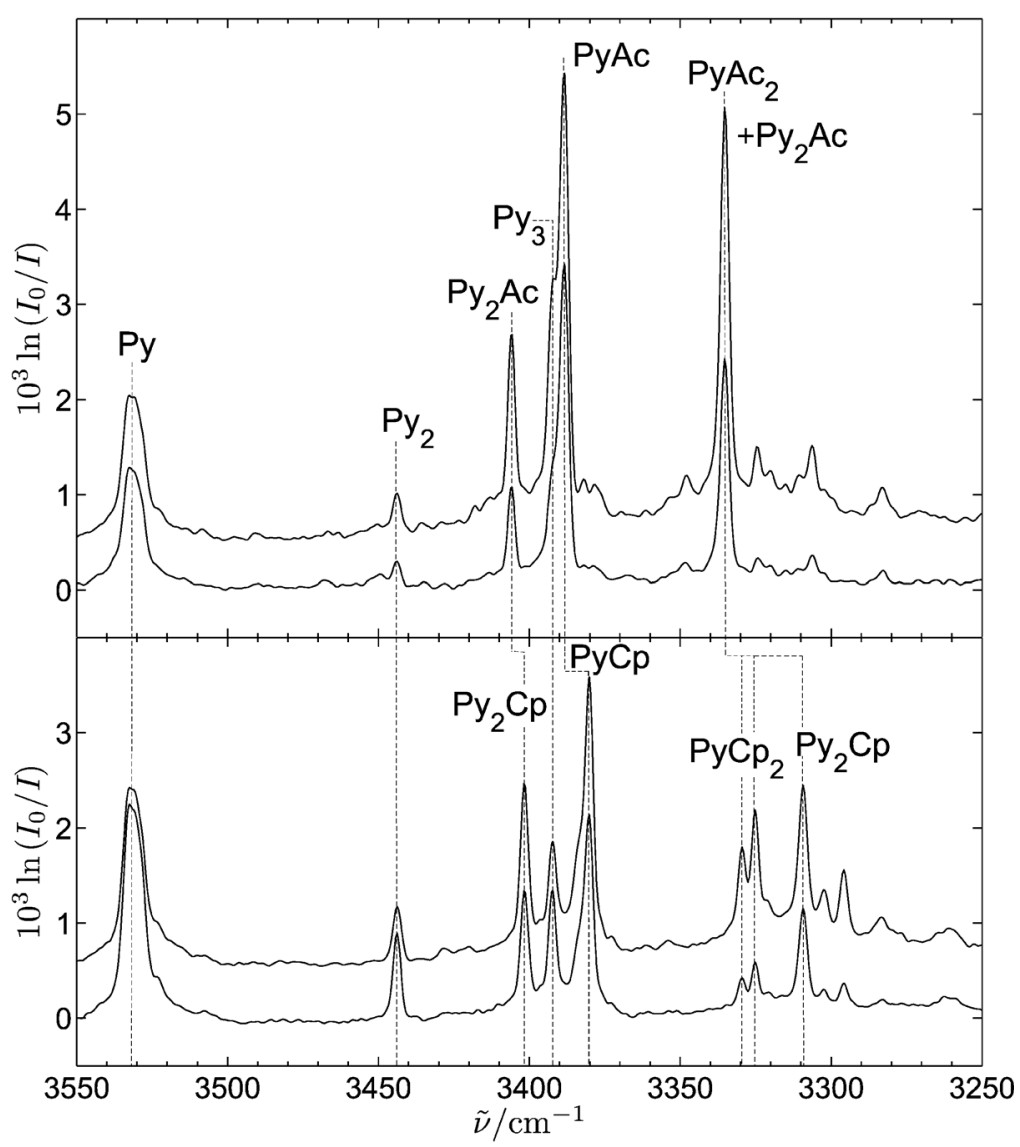

Fig. 3 FTIR jet spectra of pyrrole (Py) coexpansions with acetone (Ac, top) and cyclopentanone ( $\mathrm{Cp}$, bottom). The shift pattern when replacing acetone by the better hydrogen bond acceptor cyclopentanone shows that the mixed Py/Ac band near $3335 \mathrm{~cm}^{-1}$ involves two different species, namely PyAc 2 and the elusive ${ }^{96} \mathrm{cyclic}_{\mathrm{Py}} \mathrm{Ac}$.

pattern consisting of two $\mathrm{N}-\mathrm{H} \cdots \mathrm{O}=\mathrm{C}$ hydrogen bonds is replaced by a singly hydrogen-bonded dimer topology when one of the alanines has an inverted (D-) configuration. More generally, the spectra show that aggregation is accompanied by systematic conformational isomerization ${ }^{89}$ around the soft $\mathrm{N}-\mathrm{C}_{\alpha}$ peptide angle $\phi$.

Chiral molecules with the $\mathrm{NH}$ or $\mathrm{OH}$ functionality can reveal chirality recognition when interacting with each other. ${ }^{100-102}$ In addition to the strong hydrogen bond contacts, weaker secondary interactions are typically responsible for the discrimination between an image and a mirror image. ${ }^{103,104}$ If they are missing in a molecular complex due to geometrical constraints, no chirality recognition is observed. ${ }^{105}$ This is quite in contrast to the solid state. ${ }^{106,107}$ In other cases such as menthol, the solid state differences persist in the complexes. ${ }^{108}$ While these recognition effects are most easily understood at the level of dimers, ${ }^{8,109}$ the jet-FTIR studies have also revealed remarkable examples of chirality discrimination for higher aggregates, such as tetramers. ${ }^{110,111}$ The interplay between cooperative $\mathrm{OH} \cdots \mathrm{OH}$ bonds and more isolated $\mathrm{OH} \cdots \mathrm{O}=\mathrm{C}$ bonds is particularly subtle. ${ }^{17,112,113} \alpha$-Hydroxyesters have thus turned out to be outstandingly interesting systems in terms of aggregation, ${ }^{110,114}$ also due to the competition between inter- and intramolecular hydrogen bonds. ${ }^{17}$ Because of the high baseline stability and reproducibility of the jet-FTIR spectra, subtraction schemes can be employed to discriminate between homo- and heterochiral complexes, ${ }^{101}$ compensating somewhat for the lack of conformational selectivity of the method.

Fluorination introduces characteristic perturbations to the conformational preference of alcohol clusters, ${ }^{115-117}$ with ramifications to the solvation of biomolecules. ${ }^{118,119}$ The balance between $\mathrm{CH}$ and $\mathrm{OH}$ bonds to fluorine can be quite subtle, but relaxation studies using various carrier gases can reveal such differences in detail. ${ }^{116}$ On the other hand, $\alpha$-halogens can polarize $\mathrm{CH}$ bonds to such an extent that their hydrogen bonds become rather strong ${ }^{120}$ and the effects of hydrogen bonding on the infrared fundamental intensity can be remarkable. ${ }^{120-122}$ Less polarized $\mathrm{CH}$ bonds also undergo interactions with hydrogen bond acceptors, ${ }^{123,124}$ but it is often difficult to identify their stabilizing role in the presence of strong electrostatic forces.

In summary, the hydride stretching dynamics of jet-cooled clusters can be routinely characterized using FTIR techniques and represents a mature field in the characterization of molecular recognition phenomena via hydrogen bonds.

\section{Double and triple bonds}

Vibrations linked to hydrogen bond acceptor groups are normally far less sensitive to a hydrogen-bonded partner than the hydride donors. Therefore, their role in unraveling hydrogen bond topologies in jet-cooled clusters has often been an auxiliary one. 
The strong carbonyl stretching bands do shift to lower wavenumber when engaged into intermolecular hydrogen bonds, ${ }^{54,98}$ whereas they can shift to higher wavenumber, if competing cluster interactions break open an intramolecular hydrogen bond, such as in $\alpha$-hydroxy carbonyl compounds. ${ }^{110}$ This is a characteristic signature of changes in hydrogen bond topology. In dimers of carboxylic acids, the $\mathrm{C}=\mathrm{O}$ low frequency shift is pronounced $^{16}$ and corresponds to the onset of the transition from a double bond to a single bond upon concerted proton transfer. For isomers of the classical doubly bridged dimer structure, less pronounced red-shifts are observed. ${ }^{125}$

In the absence of classical hydrogen bond options, carbonyl groups cause and probe electrostatically driven stacking interactions ${ }^{124,126}$ which have a major influence on the volatility of lactones and cyclic carbonates. They are also sensitive to resonance with overtone and combination modes from the fingerprint region, which may be turned on and off by intermolecular interaction. ${ }^{124}$ For small molecules involving double bonds with a strong polarity change upon vibration, extended aggregation can lead to characteristic distortions of the band profile, which are related to the shape, size and order of the nanoparticles. ${ }^{127,128}$

An interesting case where a symmetry-forbidden triple bond mode acquires weak IR intensity through complexation of a neighboring atom is ammonia-acetylene. ${ }^{129}$ The less subtle case of direct complexation of $\mathrm{C}=\mathrm{C}$ bonds by hydride groups remains to be explored in the $\mathrm{C}=\mathrm{C}$-stretching fundamental range by pulsed jet-FTIR techniques, whereas it has been studied in the gas phase. ${ }^{130,131}$

\section{The fingerprint region}

An advantage of FTIR spectroscopy over laser-based techniques is that the fingerprint region is routinely available, whereas tabletop lasers are often restricted to selected narrow ranges. ${ }^{56,132}$ In fact, the early work on clusters in the $10 \mu \mathrm{m}$ range has been dominated by line-tuneable $\mathrm{CO}_{2}$ lasers. ${ }^{71,133,134}$ More recently, broadband coverage became available in this range at reasonably high resolution, ${ }^{76,135,136}$ whereas free electron lasers offer at least moderate spectral resolution. ${ }^{137}$ Conformational and size selectivity is particularly valuable ${ }^{135}$ due to the congested nature of this spectral range. However, careful examination of the stagnation pressure or concentration dependence sometimes also allows for conclusions based on fingerprint FTIR jet spectra. ${ }^{124}$ In conformationally flexible molecules, aggregation often induces a qualitative switch in the conformation, a phenomenon which may be called adaptive aggregation. ${ }^{89}$ It is omnipresent in enzyme-substrate pre-reactive complexes and while it also manifests itself in the high frequency hydride stretching range, the spectral changes in the fingerprint region are usually more pronounced. ${ }^{89,112,138}$

\section{The far infrared}

Technologically more challenging but also more rewarding is the far infrared range below about $700 \mathrm{~cm}^{-1}$. Instead of $1-\mathrm{N}_{2}$ cooled photodetectors, relatively slow He-cooled bolometers are usually employed in this region, which is characterized by weak incoherent light sources and poor transmission through optical components. Part of this can be compensated by opening the aperture of the FTIR beam. However, the low speed of bolometers requires long gas pulses or low resolution. A substantial advantage of the jet-FTIR approach is that the hydride stretching and far infrared libration bands can be studied almost simultaneously under identical expansion conditions. ${ }^{139}$ First steps into this direction were made for $\mathrm{HF}$, water and methanol using continuous $^{48}$ and seeded pulsed expansions. ${ }^{14,139}$ More recently, higher resolution and dilution could be achieved, allowing for the separation of small cluster contributions. ${ }^{140}$

Librational motions of the central hydrogen away from the hydrogen bond are very sensitive to cluster size, symmetry, bulkiness, ${ }^{141}$ bond strength and deuteration. ${ }^{139}$ Starting as hindered rotations well below $100 \mathrm{~cm}^{-1}$, they can be shifted up to $1000 \mathrm{~cm}^{-1}$ due to the hydrogen bond constraint. ${ }^{14,139,142-144}$ Thus, they have a major influence on the zero-point energy and spectral properties of a complex. ${ }^{121}$ Although the overall width of librational bands in the condensed phase and in extended clusters is very large, ${ }^{10}$ the jet measurements ${ }^{141}$ show that the isolated cluster bands can be fairly narrow. Thus, the overall width should not be blamed predominantly on fast energy redistribution out of the librational coordinate, but rather on inhomogeneous contributions due to different environments of individual molecules in the liquid. The detection of hindered translations or hydrogen bond stretching modes is considerably more challenging due to their low frequency. ${ }^{139,144}$ This is a field where Raman spectroscopy is still more powerful. ${ }^{145}$ The lowfrequency librations have also frequently been accessible by combination band spectroscopy in the mid $\operatorname{IR}^{67,146}$ or in simple cases by high resolution far infrared laser spectroscopy. ${ }^{147}$ For fluorescing systems, dispersed fluorescence provides an alternative access, with outstandingly detailed information on intermolecular vibrations for many UV-chromophores. ${ }^{148-150}$

Recently, it has become possible for the first time to extend the range of bolometric jet-FTIR spectroscopy down to $200 \mathrm{~cm}^{-1}$, allowing to fix the band center of the highest frequency hydrogen bond mode of the formic acid dimer. ${ }^{52}$ (Fig. 4). Hopefully, the coupling of jet-FTIR spectrometers with bright synchrotron sources like in the jet-AILES project ${ }^{47}$ will give access to even lower wavenumbers, as already demonstrated in gas phase studies. ${ }^{151}$ This is an arena where many important insights into the anharmonicity of librational motion can be expected in the future. Such information is essential for the description of dissociation equilibria of hydrogen-bonded complexes, ${ }^{52}$ an otherwise rather challenging endeavour. ${ }^{152,153}$ While stimulated emission pumping is very powerful in determining absolute binding energies of aromatic complexes ${ }^{154}$ due to the efficient vibrational relaxation via intermolecular modes, qualitative binding energy sequences of conformers are also accessible to FTIR jet studies, if conformational conversion is active. ${ }^{29}$

Far-infrared and sub-mm ( THz) spectra are of much current interest in relationship with modified water dynamics around peptides. ${ }^{155,156}$ In this context, it becomes clear that the 


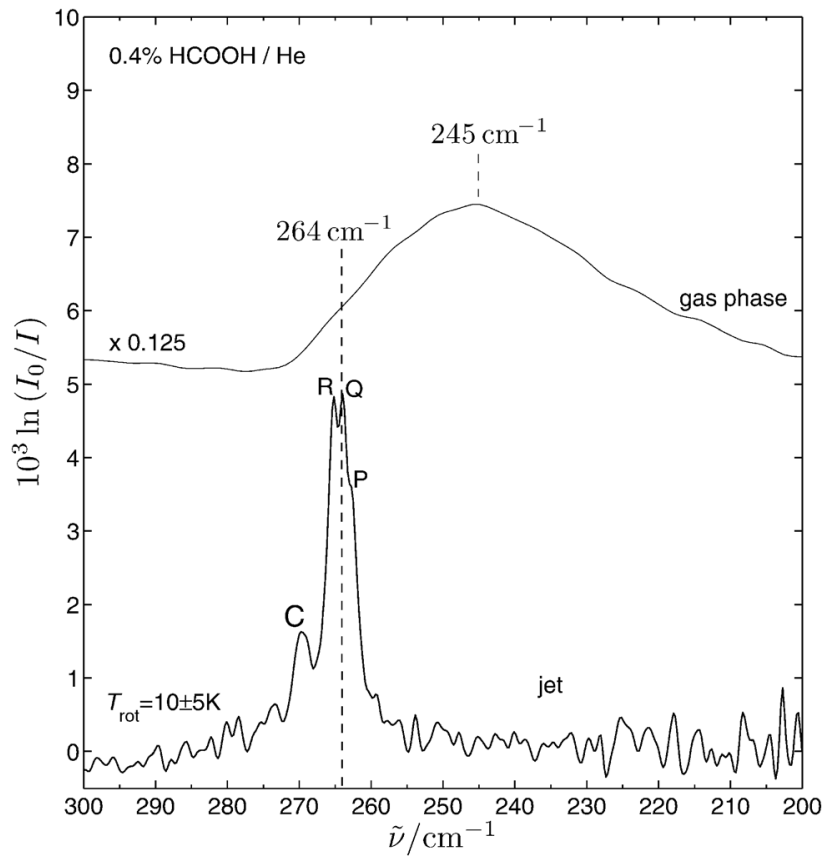

Fig. 4 Comparison of room temperature gas phase (top trace, $\approx 2 \%$ dimer fraction) and jet expansion spectra (bottom trace, $\approx 30 \%$ dimer fraction) of a $0.4 \% \mathrm{HCOOH}-\mathrm{He}$ mixture in the region of the in-plane $\mathrm{B}_{\mathrm{u}}$ hydrogen bond libration of the dimer. The thermal shift makes a band center determination at room temperature very difficult, whereas the jet spectrum is an order of magnitude more narrow, hints at a PQR structure and also shows a band due to larger clusters (C).

vibrational interpretation of spectral absorbance in solution is nontrivial. Work on model systems at low temperature may be able to contribute to a better understanding of the hydrogen bond dynamics in this range.

\section{The near infrared}

Another challenging spectral region for jet-FTIR spectroscopy is the near infrared range with its hydride stretching overtones. They are essential for an assessment of how anharmonicity changes with hydrogen bonding. ${ }^{157}$ In essence, hydrogen bonding is a kind of pre-reactive state, which can initiate chemical bondbreaking. Until now, $\mathrm{OH}$ anharmonicities in non-aromatic hydrogen-bonded molecular complexes in the gas phase usually had to be estimated from deuteration effects, ${ }^{67}$ but these rely on the absence of mode-mixing in the lower frequency range, which is often not the case in polyatomic molecules. For weak intramolecular hydrogen bonds in molecules, gas phase overtone measurements reveal such $\mathrm{OH}$ anharmonicities more directly, but it is difficult to compare with the non-hydrogenbonded reference. ${ }^{131}$ On the other hand, the IR intensity gain of $\mathrm{OH}$ stretching fundamentals in stronger hydrogen bonds is completely lost or even negative for overtones, making this particularly challenging from the experimental side. The first jet-FTIR evidence for an alcohol dimer overtone was found for trifluoroethanol, thanks to its relatively weak intermolecular hydrogen bond. ${ }^{117}$ The spectra provide valuable support for the

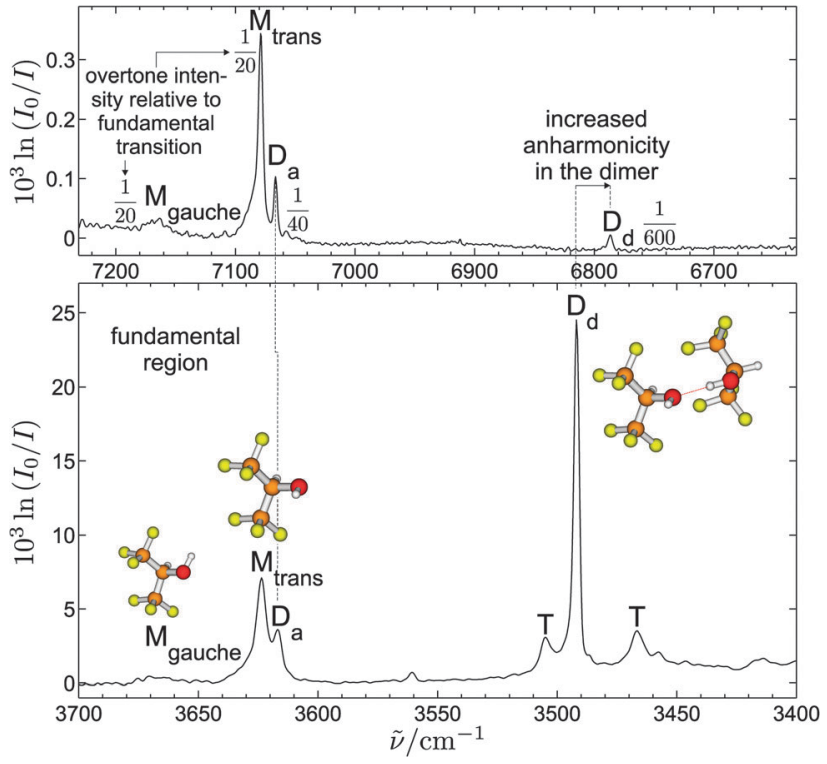

Fig. 5 Comparison of $\mathrm{OH}$ stretching fundamental and overtone spectra of HFIP monomers, dimers and trimers in terms of infrared intensity and band position under identical expansion conditions. 0.6\% HFIP in He were expanded at 0.8 bar through the $600 \times 0.2 \mathrm{~mm}^{2}$ slit nozzle. The wavenumber scales differ by a factor of exactly two and were shifted to superimpose the monomer signals to make the $18 \%$ increase in anharmonicity for the donor $\mathrm{OH}$ stretch in the dimer explicit. The drop in intensity for the overtone is already large for the monomers $(20 \times)$ and the dimer acceptor $(40 \times)$ due to their intramolecular hydrogen bonds, but even larger $(600 \times)$ for the dimer donor with its intermolecular hydrogen bond and unmeasurably large for the trimer. See the text for further explanations.

expectation that anharmonicity increases substantially for the hydrogen bond donor, in this case by $15 \%$. The first overtone of the donor $\mathrm{OH}$ is three orders of magnitude weaker than the fundamental. ${ }^{117}$ Another example is the pyrrole dimer, where some evidence for dimer overtones was found. ${ }^{86}$ This is a field where we expect to make experimental progress by optimizing the detection sensitivity and cluster density.

As an example, Fig. 5 compares the fundamental and first overtone regions for nominally identical supersonic jet expansions of 1,1,1,3,3,3-hexafluoroisopropanol (HFIP) through the $600 \mathrm{~mm}$ nozzle. The windows are scaled such that the dominant monomer transitions coincide and the overtone wavenumber scale is compressed by exactly a factor of two. This allows the observer to visually detect the increase in diagonal anharmonicity upon dimer formation. In the fundamental region, one can see the most stable monomer conformation $\mathrm{M}_{\text {trans }}$, in which the $\mathrm{OH}$-group points midway between the two $\mathrm{CF}_{3}$ groups. Only traces of the transiently chiral gauche isomer survive the expansion. The most stable dimer was previously shown to be built from two trans monomers. ${ }^{115}$ It gives rise to a strongly shifted hydrogen bond donor band near $3500 \mathrm{~cm}^{-1}$ $\left(D_{d}\right)$ and a weakly shifted hydrogen bond acceptor band $\left(D_{a}\right)$ that appear together with weaker trimer signals (T) and a very weak band near $3560 \mathrm{~cm}^{-1}$ which could be due to a gauche donor $\mathrm{OH}$ stretch, perhaps in a gauche-gauche dimer.

Anyway, all these weaker bands are not discernible in the overtone region (upper panel of Fig. 5). The trimer is not visible, 
as it profits from a large hydrogen bond-induced IR intensity gain in the fundamental region, which is lost for the twoquantum transition. ${ }^{117}$ To a lesser extent, this is also true for the dimer. While the monomer intensity drops by a factor of 20 upon overtone excitation, the weakly enhanced $\mathrm{OH} \cdots \mathrm{F}$ hydrogen bond of the acceptor already doubles the intensity loss $(\times 40)$ and the donor hydrogen bond leads to an overtone intensity drop by a factor of 600 relative to the fundamental. One should note that the 20 -fold monomer intensity drop is already more than one would expect from a simple alcohol $\mathrm{OH}$ stretching overtone. The reason is the weak $\mathrm{OH} \cdots \mathrm{F}$ contact which exists in both monomer conformations, similar to trifluoroethanol. ${ }^{117}$ The overtone intensity drop relative to the fundamental is thus seen to be a very sensitive indicator of the hydrogen bond strength. This makes a linear technique such as FTIR, which is able to probe both regions with reliable relative intensities, particularly valuable.

Concerning the diagonal anharmonicity constant $\omega_{e} x_{e}$ of the localized $\mathrm{OH}$ oscillator (in diatomic molecule notation ${ }^{67}$ ), the spectra show that it increases from $84 \pm 1 \mathrm{~cm}^{-1}$ for the two monomer conformations and $\mathrm{D}_{\mathrm{a}}$ to $99 \mathrm{~cm}^{-1}$ for $\mathrm{D}_{\mathrm{d}}$. This moderate $18 \%$ increase in anharmonicity is to be contrasted to the $3000 \%$ intensity effect. We aim for a systematic study of such potential and dipole hypersurface effects in alcohol clusters by directly comparing fundamental and overtone spectra in the same experimental setup.

It should be noted that fs $2 \mathrm{D}$ IR spectroscopy in solution ${ }^{158}$ provides an elegant access to the first overtone by monitoring excited state absorption after population of the fundamental. ${ }^{159}$ However, solution and thermal shifts limit the usefulness for accurate determinations and quantum-chemical modeling of anharmonicity constants. Furthermore, it must be emphasized that the popular approach to extract $\mathrm{OH}$-stretching anharmonicity effects in liquids from classical molecular dynamics simulations ${ }^{160}$ is somewhat misleading. To reproduce the stretching fundamental of a simple Morse oscillator with harmonic wavenumber $\omega_{\mathrm{e}}$, one must use a temperature $T$ on the order of $h c \omega_{\mathrm{e}} / k$. For the first overtone, the required temperature is even $50 \%$ higher. This is clearly 1-2 orders of magnitude too high for hydride stretching modes under ambient conditions, such that molecular dynamics simulations only reproduce a small fraction of their pertinent diagonal anharmonicity.

In principle, overtones of hydrogen-bonded complexes should be universally accessible at much higher sensitivity by nonresonant UV ionization of laser-excited overtone states. ${ }^{161,162}$ Besides potential complications by dynamics in the ionic state of hydrogen-bonded clusters and non-linear intensity effects, it seems that this technique has predominantly been applied to aromatic monomers, ${ }^{162,163}$ but not to simple alcohol clusters. For aromatic systems, resonant IR-UV is of course also an attractive option. Furthermore, if band strength is not an issue, one can exploit saturation and detect very weak vibrational transitions. ${ }^{164}$ Higher overtones are nevertheless better probed by laser experiments, e.g. cavity ring down techniques, ${ }^{165,166}$ intracavity photoacoustics, ${ }^{131}$ non-resonant ionization ${ }^{163}$ and action spectroscopy. ${ }^{167,168}$ This is also true for first overtone jet spectroscopy of small molecules, where rotational resolution can be helpful. ${ }^{169-174}$

\section{Comparisons}

In this chapter, the femtisecond single-mole pulsed jet approach shall be compared to a selection of other spectroscopic techniques which aim at a characterization of hydrogen bond vibrations, with some emphasis on advantages, synergies and limitations.

\subsection{Comparison to gas phase measurements near room temperature}

Some hydrogen bond precursors can form thermodynamically stable dimers or even oligomers in the gas phase. The latter is the case for $\mathrm{HF},{ }^{14}$ methanol, ${ }^{10}$ and a few other ring-aggregating molecules, ${ }^{32,84}$ the reason being a small driving force for further aggregation. Carboxylic acids are even more hierarchically aggregating with very little driving force beyond the dimer. ${ }^{16,52}$ In other systems, dimers can be observed in the spectrum even in low abundance because the monomers have a weak fundamental band strength. ${ }^{91,120-122}$ In all cases, the band maxima observed near room temperature are strongly shifted compared to the $0 \mathrm{~K}$ band origin and considerably broadened (see Fig. 4). The reason is a population of excited intermolecular modes, which weaken the hydrogen bond and shift the average transition towards that of the monomer. In hydride stretching modes, this is the isolated stretch (typically found at higher frequency), whereas in hydrogen bond librations, the isolated limit is a free rotation or torsion (to lower frequency). As a rule of thumb, the thermal shift of the band maximum is not too far from one full width at half maximum of the thermally broadened band, i.e. the true band center is close to the onset of the thermal band on the monomer-averted side. The width itself can reach the same order of magnitude as the complexation shift, if the available thermal energy is comparable to the hydrogen bond strength. The simplest of all models would relate a small thermal wavenumber shift $\Delta \tilde{\nu}_{\text {th }}$ of the band maximum from the low temperature limit to the ratio of the thermal energy $R T$ to the hydrogen bond dissociation energy $D_{0}$ via the hydrogen bond-induced interaction shift under jet conditions $\Delta \tilde{\nu}_{0}$ and some proportionality factor $s$ :

$$
\Delta \tilde{\nu}_{\mathrm{th}}=-s \Delta \tilde{\nu}_{0} \frac{R T}{D_{0}}
$$

The - sign implies that thermal shifts usually counteract the interaction shifts. For systems as different as the methanol dimer, ${ }^{10}$ chloroform-ammonia, ${ }^{120}$ and the librational mode of the formic acid dimer, ${ }^{52} s$ values on the order of 1 are obtained, depending on the value of $D_{0}$. Where the interaction is less dominated by hydrogen bonding, substantially larger values for $s$ may be expected.

Neglecting thermal shift and broadening in molecular complexes can have consequences for the determination of partition functions ${ }^{52}$ or for the comparison of complexation shifts to theory. Furthermore, it has led to an erroneous assignment of 
an atmospheric near infrared absorption signal to a water dimer overtone. ${ }^{175}$ If the infrared intensity changes much upon complexation, ${ }^{88}$ it also affects estimates of the dimerization equilibrium constant, ${ }^{115}$ because thermally excited states will typically fall short of the complexation change predicted at $0 \mathrm{~K}$.

\subsection{Comparison to size- and conformationally selective methods}

The combination of jet-FTIR spectroscopy with size-selective techniques relying on molecular beam scattering ${ }^{133}$ has helped in confirming size assignments based on stagnation pressure dependence and in detecting cluster heating effects. ${ }^{176,177}$ Non-rigorous variants of such size-selective techniques ${ }^{178}$ can however also be misleading ${ }^{13}$ and cross-checking by independent methods is always of advantage. ${ }^{179}$ UV-IR double resonance requires the presence of a suitable UV chromophore, typically an aromatic substituent, ${ }^{102}$ which reduces the volatility. Therefore, routine overlap with jet-FTIR techniques only became possible with the development of a heated nozzle for the latter. ${ }^{34}$ The case of aromatic methyl mandelate dimers illustrates the complementarity between the two approaches. ${ }^{94}$ Remarkably, the interaction between the phenyl rings in the heterochiral pair is strong enough to prevent a second intermolecular $\mathrm{OH} \cdots \mathrm{O}=\mathrm{C}$ hydrogen bond to be formed. The resulting dimer is more strongly bound than the corresponding homochiral dimer with two strong hydrogen bonds. With the improvement of the sensitivity of heated nozzle jet-FTIR spectroscopy, it should be possible to investigate more aromatic systems with direct absorption techniques. Normally, one expects similar conformational preferences despite the rather different expansion conditions in free jets and skimmed molecular beams. In the complex of 4-phenyl-imidazole with water, the counterintuitive exclusive observation by UV-IR double resonance ${ }^{180}$ of water as a hydrogen bond acceptor may have to be complemented by the hydrogen bond donor isomer, which is found predominantly when combining imidazole with water in an FTIR study. ${ }^{29}$ While UV-IR techniques offer powerful sensitivity, selectivity ${ }^{181}$ and time-resolution ${ }^{182}$ as well as frequency coverage, ${ }^{76,135}$ complementary FTIR studies can sometimes be helpful to complete the picture. ${ }^{95}$ Although the conformational selectivity of selective methods can be switched off $^{183}$ towards a more panoramic viewpoint, aspects of action spectroscopy usually persist and a comparison to linear infrared intensities from direct absorption may be instructive.

However, the main field of application for direct absorption spectroscopy will probably remain that of systems without a well-behaved UV chromophore. While such systems can always be tagged with a suitable chromophore to allow for UV/IR double resonance, a careful analysis or a sufficiently large system/tag ratio is required to ensure that the tag does not influence the system. ${ }^{184-186}$ The use of VUV techniques to study the IR spectra of tag-free systems with double-resonance strategies $^{187}$ often requires a careful disentanglement of fragmentation channels. ${ }^{68}$ This is also true for IR-modulated photoionization of sodium on hydrogen-bonded clusters. ${ }^{188}$ $E . g$., fragmentation may have been underestimated in a recent study of sodium doped water trimers. ${ }^{189}$ Fragmentation should of course always be kept in mind when detecting hydrogenbonded systems via ionization due to the major rearrangements caused by the introduction of a charge. ${ }^{63,190}$

Microwave spectroscopy may also be considered to be sizeand conformationally selective, if the structure of the cluster can be derived. ${ }^{191,192}$ It shares with jet-FTIR techniques the absence of a UV chromophore requirement ${ }^{193}$ and often provides definitive structural answers, where vibrational spectroscopy has to rely on a comparison between predicted and observed spectra. However, it fails for non-polar structures, such as highly symmetric clusters. ${ }^{123,124,194}$ By breaking the symmetry in subtle ways, very useful information can still be extracted on the structure and dynamics of near-symmetric systems. ${ }^{195}$ The revolutionary new broadband microwave technique based on chirped pulses ${ }^{196}$ is likely to open up even wider fields of application than the broadband FTIR approach for cluster dynamics. In both cases, the ability to oversee a wide spectral range at the same time is part of the success. Apart from this multiplex advantage, both techniques share the use of large (or multiple) nozzles as a way of improving the signalto-noise ratio or reducing the substance consumption.

\subsection{Comparison to Raman spectroscopy}

In a situation of high symmetry, spontaneous Raman scattering can provide crucial assignment aids. ${ }^{197}$ In combination with FTIR spectra, it allows us to identify Davydov or vibrationally excitonic splittings between equivalent local oscillators. ${ }^{67,95} \mathrm{We}$ note that these splittings are usually in rather good agreement with harmonic predictions from theory, whereas in the electronic counterpart, ${ }^{198}$ redistribution of the oscillator strength over several Franck-Condon active modes can cause dramatic quenching. It is conceivable that such a situation also occurs in vibrational spectra involving coupled oscillators, if there is strong intensity redistribution to combination bands with low frequency modes. The combination of Raman and IR spectra helps us to assign tunneling splittings ${ }^{73,199}$ and hot bands from other low frequency modes. ${ }^{90}$ Furthermore, Raman spectra give direct access to symmetric low frequency hydrogen bond modes, ${ }^{145}$ whose antisymmetric IR active counterparts are much more difficult to observe. ${ }^{52}$

\subsection{Comparison to solid and liquid rare gas isolation}

In the early pulsed slit jet-FTIR experiments, ${ }^{10}$ it was observed that vibrational bands of $\mathrm{HCl}$ clusters would red-shift when the expansion was sufficiently dilute and He was replaced by $\mathrm{Ar}$ as a carrier gas. This phenomenon was found to be rather general, ${ }^{200}$ and the new band positions often agreed remarkably well with those of bulk Ar matrix-isolated clusters. QM/MM modeling confirmed that Ar shells form around the hydrogenbonded aggregates. ${ }^{66}$ Other than in bulk matrices, site splittings are not observed and other than in liquid Ar, lower temperatures and more narrow band profiles are typically achieved. Ar-coated water clusters have been studied by pulsed ${ }^{16}$ and continuous jet-FTIR approaches. ${ }^{50}$ Obviously, bulk matrix isolation is a much more sensitive technique than this $\mathrm{Ar}$ 
nanomatrix isolation, ${ }^{201}$ because the residence time is many orders of magnitude longer. This allows for laser hole-burning and isomerization experiments, which are not yet feasible in the jet-FTIR approach. ${ }^{138}$ Nevertheless, the ability to switch between vacuum-isolated and matrix-isolated conditions in the same experimental setup, a marriage between supersonic jets and matrix isolation, ${ }^{202}$ is of interest. From the Ar coating shift anomaly in the ethanol dimer, one can infer that the preferred conformation under vacuum is different from that in an $\mathrm{Ar}$ matrix. ${ }^{69}$ Tunneling splittings resulting from interconversion between equivalent minima on the potential energy hypersurface can be efficiently quenched in matrices, assisting in their assignment. ${ }^{199,203,204}$ In the case of pyrrole aggregates, a close comparison between bulk ${ }^{205}$ and nanomatrices ${ }^{86}$ is possible. It is also of interest to follow the Ar coating step by step, ${ }^{206}$ although this typically requires high spectral resolution or other ways of size selection. ${ }^{207,208}$

Beyond the Ar nanocoating effect, matrix isolation and jetFTIR studies provide nicely complementary information. ${ }^{144,201,209}$ Pulsed jets have also been used to prepare clusters in bulk matrices. ${ }^{210,211}$ Matrix isolation allows for photochemical isomerization processes, ${ }^{212-214}$ whereas jet-FTIR can detect metastable aggregates. ${ }^{112}$ Some motion is possible in solid quantum matrices, ${ }^{215}$ but like the supersonic jet preparation, a matrix remains largely a non-equilibrium environment. In contrast, studies of weakly bound complexes in liquid rare gas solutions $^{121,122}$ yield thermodynamic equilibrium data for clusters. The solvent shifts are temperature-dependent and can be identified by comparison to the free jet data. This may be used to improve solute-solvent models. ${ }^{122}$ Even liquid helium can show sizeable solvent shifts, ${ }^{29}$ in particular if the hydrogen bond induced shifts are large. ${ }^{216}$ On the other hand, very small hydrogen bond shifts can be reversed in sign as a function of temperature and rare gas. ${ }^{121}$

A special case of rare gas isolation is the assembly of clusters in liquid He nanodroplets. ${ }^{202,217}$ Using pulsed ${ }^{218}$ or more typically continuous expansions, ${ }^{219}$ one can generate clusters within the nanodroplets step-by-step, often favoring unstable structures due to the rapid cooling. ${ }^{219}$ Field alignment experiments ${ }^{81}$ allow for insights into the polarity and structure of the clusters and systematic pick-up studies provide absolute infrared intensities. ${ }^{220}$

The ultimate goal of the molecular cluster strategy is to approach and understand condensed matter step by step. A virtue of IR spectroscopy is that it provides access to all states of matter. However, its limited spatial resolution ${ }^{221}$ prevents the routine study of mesoscopic aggregates. Nevertheless, changes in hydrogen bond topology from dimers to glassy and crystalline solids can be followed. ${ }^{201}$

\section{Outlook}

Important challenges for future jet-FTIR studies include the extension into the very far infrared range, ${ }^{139}$ where weak translational bands meet low photon levels, and into the near infrared, ${ }^{117}$ where the band strengths are extremely small.
Using focal plane array detectors, ${ }^{221}$ it will become possible to directly image a supersonic jet expansion in terms of spatial cluster distribution or shock waves. Presently, this is restricted to a pointwise scanning mode. ${ }^{10,222}$ When the molecules become larger and more strongly interacting, heated nozzles are indispensable. Currently, heating is realized resistively, making necessary a sufficient thermal stability of the compounds. It will be somewhat challenging to combine jet-FTIR spectroscopy with laser evaporation of samples, because of the mismatch in the duty cycle between typical pulsed lasers and the much slower gas pulses. Different variants of heated nozzles should help in improving our understanding of conformational relaxation in supersonic jets. Its dependence on nozzle geometry and distance, carrier gas, barrier height and effective mass of the rearranging subunits is still not well understood, even concerning the dominant mechanisms. ${ }^{223-225}$ An attractive option is to have a sequence of alternating nozzles fed by two different compounds, ${ }^{119}$ allowing to study and possibly suppress reactive processes such as isotope exchange. ${ }^{144}$ Whether or not the large quantities of molecular clusters and nanoparticles produced in the high-throughput nozzles can be deposited on surfaces ${ }^{226}$ in a useful way remains to be seen. The use of nontraditional, broadband coherent light sources in FTIR, possibly coupled with cavity enhancement techniques, may bring advantages in particular at higher resolution. ${ }^{227}$ As it stands, the technique is a profoundly low-resolution approach and can therefore contribute only in a very indirect way to fundamental questions such as that of parity violation in molecular systems. ${ }^{228}$ However, aspects of chirality have been among the most interesting applications. ${ }^{102}$

\section{Acknowledgements}

The co-workers in the field of pulsed jet-FTIR spectroscopy are cited in the references. Their contributions are gratefully acknowledged, as is the support by the Fonds der Chemischen Industrie and the German Research Foundation over the last decade (SFB 357, SFB 602, GRK 782, Su121/4-1). We are indebted to the mechanics, glass and electronics workshops of the institute, who contributed vitally to the construction of the high throughput nozzles, and to N. Borho, who developed and characterized the first version of the filet-jet setup. R. Wugt Larsen is acknowledged for advancing the far infrared capabilities, whereas D. J. Nesbitt and M. Fárník have been instrumental in improving performance in the mid- to near-infrared. Last but not least, M. Quack has always strongly promoted the use of jet-FTIR spectroscopy in hydrogen-bonded cluster research and has generously supported the first attempts to scale it up to a powerful routine technique based on pulsed operation.

\section{References}

1 R. E. Miller, The vibrational spectroscopy and dynamics of weakly bound neutral clusters, Science, 1988, 240, 447-453.

2 T. J. Balle and W. H. Flygare, Fabry-Perot cavity pulsed Fourier transform microwave spectrometer with a pulsed nozzle particle source, Rev. Sci. Instrum., 1981, 52, 33-45. 
3 T. S. Zwier, Laser probes of conformational isomerization in flexible molecules and complexes, J. Phys. Chem. A, 2006, 110, 4133-4150.

4 D. R. Miller, Free jet sources, in Atomic and Molecular Beam Methods, ed. G. Scoles, University Press, Oxford, 1988, ch. 2, pp. 14-53.

5 G. M. Florio, E. L. Sibert III and T. S. Zwier, Fluorescencedip IR spectra of jet-cooled benzoic acid dimer in its ground and first excited singlet states, Faraday Discuss., 2001, 118, 315-330.

6 D. J. Nesbitt, High-resolution, direct infrared laser absorption spectroscopy in slit supersonic jets: intermolecular forces and unimolecular vibrational dynamics in clusters, Annu. Rev. Phys. Chem., 1994, 45, 367-399.

7 D. J. Nesbitt, High-resolution infrared spectroscopy of weakly bound molecular complexes, Chem. Rev., 1988, 88, 843-870.

8 K. Le Barbu, F. Lahmani and A. Zehnacker-Rentien, Formation of hydrogen-bonded structures in jet-cooled complexes of a chiral chromophore studied by IR/UV double resonance spectroscopy: diastereomeric complexes of \pm -2-naphtyl-1-ethanol with \pm -2-amino-1-propanol, J. Phys. Chem. A, 2002, 106, 6271-6278.

9 M. Quack, Spectra and dynamics of coupled vibrations in polyatomic molecules, Annu. Rev. Phys. Chem., 1990, 41, 839-874.

10 T. Häber, U. Schmitt and M. A. Suhm, FTIR-spectroscopy of molecular clusters in pulsed supersonic slit-jet expansions, Phys. Chem. Chem. Phys., 1999, 1, 5573-5582.

11 M. Herman, R. Georges, M. Hepp and D. Hurtmans, High resolution Fourier transform spectroscopy of jet-cooled molecules, Int. Rev. Phys. Chem., 2000, 19, 277-325.

12 D. L. Snavely, S. D. Colson and K. B. Wiberg, Rotational cooling in a supersonic expansion of ammonia, J. Chem. Phys., 1981, 74, 6975-6976.

13 D. Luckhaus, M. Quack, U. Schmitt and M. A. Suhm, On FTIR spectroscopy in asynchronously pulsed supersonic jet expansions and on the interpretation of stretching spectra of HF clusters, Ber. Bunsen-Ges. Phys. Chem., 1995, 99, 457-468.

14 M. A. Suhm, HF vapor, Ber. Bunsen-Ges. Phys. Chem., 1995, 99, 1159-1167.

15 M. Quack, U. Schmitt and M. A. Suhm, FTIR spectroscopy of hydrogen fluoride clusters in synchronously pulsed supersonic jets: isotopic isolation, substitution and 3-d condensation, Chem. Phys. Lett., 1997, 269, 29-38.

16 T. Häber, U. Schmitt, C. Emmeluth and M. A. Suhm, Ragout-jet FTIR spectroscopy of cluster isomerism and cluster dynamics: from carboxylic acid dimers to $\mathrm{N}_{2} \mathrm{O}$ nanoparticles, Faraday Discuss. Chem. Soc., 2001, 118, 331-359. +contributions to the discussion on pp. 53, 119, 174-175, 179-180, 304-309, 361-363, 367-370.

17 N. Borho, M. A. Suhm, K. Le Barbu-Debus and A. Zehnacker, Intra- $v s$. intermolecular hydrogen bonding: dimers of alpha-hydroxyesters with methanol, Phys. Chem. Chem. Phys., 2006, 8, 4449-4460.
18 A. Amrein, M. Quack and U. Schmitt, High resolution interferometric Fourier transform infrared absorption spectroscopy in supersonic free jet expansions. A new technique for ultracold gaseous samples, Z. Phys. Chem., Neue Folge, 1987, 154, 59-72.

19 J. A. Barnes and T. E. Gough, Fourier transform infrared spectroscopy of supersonic jets: observation of clusters containing sulphur hexafluoride, Chem. Phys. Lett., 1986, 130(4), 297-300.

20 J. A. Barnes and T. E. Gough, Fourier transform infrared spectroscopy of molecular clusters: the structure and internal mobility of clustered carbon dioxide, J. Chem. Phys., 1987, 86, 6012-6017.

21 C. M. Lovejoy and D. J. Nesbitt, Slit pulsed valve for generation of long-path-length supersonic expansion, Rev. Sci. Instrum., 1987, 58, 807-811.

22 K. Liu, M. G. Brown, J. D. Cruzan and R. J. Saykally, Vibration-rotation tunneling spectra of the water pentamer: Structure and dynamics, Science, 1996, 271, 62-64.

23 J. Ballard, D. Newnham and M. Page, A novel cryogenic molecular beam. Application to supersonic jet spectroscopy of gases, Chem. Phys. Lett., 1993, 208, 295-298.

24 D. McNaughton, D. McGilvery and E. G. Robertson, Highresolution FTIR-Jet spectroscopy of $\mathrm{CCl}_{2} \mathrm{~F}_{2}$, J. Chem. Soc., Faraday Trans., 1994, 90, 1055-1060.

25 U. Even, J. Jortner, D. Noy, N. Lavie and C. Cossart-Magos, Cooling of large molecules below $1 \mathrm{~K}$ and He clusters formation, J. Chem. Phys., 2000, 112, 8068-8071.

26 S. Hirabayashi and Y. Hirahara, Step-scan Fourier transform infrared absorption spectroscopy of acetylene monomer and solid in a supersonic free jet, Chem. Phys. Lett., 2002, 361, 265-270.

27 S. Hirabayashi, N. Yazawa and Y. Hirahara, Step-scan Fourier-transform infrared absorption spectroscopy of cubic solid acetylene clusters, J. Phys. Chem. A, 2003, 107, 4829-4833.

28 A. E. Beylich, Struktur von Ueberschall-Freistrahlen aus Schlitzblenden, Z. Flugwiss. Weltraumforsch., 1979, 3, 48-58.

29 J. Zischang, J. J. Lee and M. A. Suhm, Communication: Where does the first water molecule go in imidazole?, J. Chem. Phys., 2011, 135, 061102.

30 C. Emmeluth, V. Dyczmons and M. A. Suhm, Tuning the hydrogen bond donor/acceptor isomerism in jet-cooled mixed dimers of aliphatic alcohols, J. Phys. Chem. A, 2006, 110, 2906-2915.

31 S. Tanimura, Y Okada and K Takeuchi, FTIR spectroscopy of $\mathrm{UF}_{6}$ clustering in a supersonic Laval nozzle, J. Phys. Chem., 1996, 100, 2842-2848.

32 C. A. Rice, N. Borho and M. A. Suhm, Dimerization of pyrazole in slit jet expansions, Z. Phys. Chem., 2005, 219, 379-388.

33 M. Albrecht, P. Zielke, C. A. Rice and M. A. Suhm, Variations of bite angle and coupling patterns in double hydrogen bonds: the case of oxime dimers, J. Mol. Struct., 2008, 880, 2-13. 
34 C. Cézard, C. A. Rice and M. A. Suhm, OH-stretching redshifts in bulky hydrogen-bonded alcohols: jet spectroscopy and modeling, J. Phys. Chem. A, 2006, 110, 9839-9848.

35 A. Bonnamy, D. Hermsdorf, R. Ueberschaer and R. Signorell, Characterization of the rapid expansion of supercritical solutions by Fourier transform infrared spectroscopy in situ, Rev. Sci. Instrum., 2005, 76, 053904.

36 C. H. Sin, M. R. Linford and S. R. Goates, Supercritical fluid/supersonic jet spectroscopy with a sheath-flow nozzle, Anal. Chem., 1992, 64, 233-238.

37 W. Christen, S. Geggier, S. Grigorenko and K. Rademann, Pulsed supersonic expansion of nonvolatile solids, Rev. Sci. Instrum., 2004, 75, 5048-5049.

38 T. Lenzer, R. Bürsing, A. Dittmer, S. S. Panja, D. A. Wild and K. Oum, Rotational and vibrational cooling in pulsed high-pressure molecular beam expansions from 3 bar into the supercritical regime, J. Phys. Chem. A, 2010, 114, 6377-6383.

39 H. Laksmono, S. Tanimura, H. C. Allen, G. Wilemski, M. S. Zahniser, J. H. Shorter, D. D. Nelson, J. Barry McManus and B. E. Wyslouzil, Monomer, clusters, liquid: an integrated spectroscopic study of methanol condensation, Phys. Chem. Chem. Phys., 2011, 13, 5855-5871.

40 A. Bonnamy, R. Georges, A. Benidar, J. Boissoles, A. Canosa and B. R. Rowe, Infrared spectroscopy of $\left(\mathrm{CO}_{2}\right)_{N}$ nanoparticles $(30<N<14500)$ flowing in a uniform supersonic expansion, J. Chem. Phys., 2003, 118, 3612-3621.

41 A. Bonnamy, R. Georges, E. Hugo and R. Signorell, IR signature of $\left(\mathrm{CO}_{2}\right)_{N}$ clusters: size, shape and structural effects, Phys. Chem. Chem. Phys., 2005, 7, 963-969.

42 I. R. Sims, J. L. Queffelec, A. Defrance, C. Rebrion-Rowe, D. Travers, B. R. Rowe and I. W. M. Smith, Ultra-low temperature kinetics of neutral-neutral reactions: the reaction $\mathrm{CN}+\mathrm{O}_{2}$ down to $26 \mathrm{~K}, \mathrm{~J}$. Chem. Phys., 1992, 97, 8798-8800.

43 M. Herman, K. Didriche, D. Hurtmans, B. Kizil, P. Macko, A. Rizopoulos and P. Van Poucke, FANTASIO: a versatile experimental set-up to investigate jet-cooled molecules, Mol. Phys., 2007, 105, 815-823.

44 R. F. Meads, A. L. McIntosh, J. I. Arno, C. L. Hartz, R. R. Lucchese and J. W. Bevan, Rovibrationally resolved, Fourier-transform near infrared absorption spectroscopy of the $\nu_{1}$ and $\nu_{2}$ vibrations of the $\mathrm{HCl}$ dimer in a supersonic jet, J. Chem. Phys., 1994, 101, 4593-4598.

45 C. L. Hartz, B. A. Wofford, R. F. Meads, R. R. Lucchese and J. W. Bevan, A near-infrared Fourier-transform planar supersonic jet spectrometer for rovibrational studies of weakly bound dimers and trimers, Rev. Sci. Instrum., 1995, 66, 4375-4384.

46 R. Georges, M. Bach and M. Herman, High resolution FTIR spectroscopy using a slit jet: sampling the overtone spectrum of ${ }^{12} \mathrm{C}_{2} \mathrm{H}_{4}$, Mol. Phys., 1997, 90, 381-387.

47 M. Cirtog, P. Asselin, P. Soulard, B. Tremblay, B. Madebène, M. E. Alikhani, R. Georges, A. Moudens, M. Goubet, T. R. Huet, O. Pirali and P. Roy, The $\left(\mathrm{CH}_{2}\right)_{2} \mathrm{O}-$ $\mathrm{H}_{2} \mathrm{O}$ hydrogen bonded complex. Ab initio calculations and
Fourier transform infrared spectroscopy from neon matrix and a new supersonic jet experiment coupled to the infrared AILES beamline of synchrotron SOLEIL, J. Phys. Chem. A, 2011, 115, 2523-2532.

48 L. M. Goss, S. W. Sharpe, T. A. Blake, V. Vaida and J. W. Brault, Direct absorption spectroscopy of water clusters, J. Phys. Chem. A, 1999, 103, 8620-8624.

49 R. Georges, M. Freytes, D. Hurtmans, I. Kleiner, J. Vander Auwera and M. Herman, Jet-cooled and room temperature FTIR spectra of the dimer of formic acid in the gas phase, Chem. Phys., 2004, 305, 187-196.

50 A. Moudens, R. Georges, M. Goubet, J. Makarewicz, S. E. Lokshtanov and A. A. Vigasin, Direct absorption spectroscopy of water clusters formed in a continuous slit nozzle expansion, J. Chem. Phys., 2009, 131, 204312.

51 D. Luckhaus and M. Quack, High-resolution FTIR spectra of $\mathrm{NO}_{2}$ and $\mathrm{N}_{2} \mathrm{O}_{4}$ in supersonic jet expansions and their rovibrational analysis, Chem. Phys. Lett., 1992, 199, 293-301.

52 F. Kollipost, R. Wugt Larsen, A. V. Domanskaya, M. Nörenberg and M. A. Suhm, Communication: the highest frequency hydrogen bond vibration and an experimental value for the dissociation energy of formic acid dimer, J. Chem. Phys., 2012, 136, 151101.

53 K. Liu, R. S. Fellers, M. R. Viant, R. P. McLaughlin, M. G. Brown and R. J. Saykally, A long path length pulsed slit valve appropriate for high temperature operation: infrared spectroscopy of jet-cooled large water clusters and nucleotide bases, Rev. Sci. Instrum., 1996, 67, 410-416.

54 M. Albrecht, C. A. Rice and M. A. Suhm, Elementary peptide motifs in the gas phase: FTIR aggregation study of formamide, acetamide, $N$-methylformamide and $N$-methylacetamide, J. Phys. Chem. A, 2008, 112, 7530-7542.

55 G. Gimmler and M. Havenith, Free-jet infrared diode laser spectroscopy of the $\nu_{2}$-band of the $\mathrm{Ar}-\mathrm{N}_{2} \mathrm{O}$ van der Waals complex, J. Mol. Struct., 2001, 599, 117-123.

56 V. Boudon, M. Rotger, Y. He, H. Hollenstein, M. Quack and U. Schmitt, High-resolution spectroscopy of the $\nu_{3}$ band of $\mathrm{WF}_{6}$ and $\mathrm{ReF}_{6}$ in a supersonic jet, J. Chem. Phys., 2002, 117, 3196-3207.

57 W. S. Tam, I. Leonov and Y. Xu, Pulsed slit jet cavity ringdown spectroscopy with a midinfrared lead salt diode laser, Rev. Sci. Instrum., 2006, 77, 063117.

58 Ö. Birer and M. Havenith, High-resolution infrared spectroscopy of the formic acid dimer, Annu. Rev. Phys. Chem., 2009, 60, 263-275.

59 A. Dehghany, A. R. W. McKellar, M. Afshari and N. Moazzen-Ahmadi, High-resolution infrared spectroscopy of carbon dioxide dimers, trimers and larger clusters, Mol. Phys., 2010, 108, 2195-2205.

60 P. Asselin, P. Soulard, B. Madebene, M. E. Alikhani and M. Lewerenz, Vibrational dynamics of the hydrogen bond in $\mathrm{H}_{2} \mathrm{~S}-\mathrm{HF}$ : Fourier-transform-infrared spectra and $a b$ initio theory, Phys. Chem. Chem. Phys., 2006, 8, 1785-1793.

61 U. Buck and F. Huisken, Infrared spectroscopy of sizeselected water and methanol clusters, Chem. Rev., 2000, 100, 3863-3890. 
62 E. Gloaguen, R. Pollet, F. Piuzzi, B. Tardivel and M. Mons, Gas phase folding of an (Ala $)_{4}$ neutral peptide chain: spectroscopic evidence for the formation of a $\beta$-hairpin H-bonding pattern, Phys. Chem. Chem. Phys., 2009, 11, 11385-11388.

63 J. M. Lisy, A. Tramer, M. F. Vernon and Y. T. Lee, Vibrational predissociation spectra of $(\mathrm{HF})_{n}, n=2-6, \mathrm{~J}$. Chem. Phys., 1981, 75(9), 4733-4734.

64 B. J. Howard, T. R. Dyke and W. Klemperer, The molecular beam spectrum and the structure of the hydrogen fluoride dimer, J. Chem. Phys., 1984, 81(12), 5417-5425.

65 T. Häber, FTIR-spectroscopy of isolated and argon coated $(\mathrm{HBr})_{n \leq 4}$ clusters in supersonic jets, Phys. Chem. Chem. Phys., 2003, 5, 1365-1369.

66 A. V. Bochenkova, M. A. Suhm, A. A. Granovsky and A. V. Nemukhin, Hybrid diatomics-in-molecules-based quantum mechanical/molecular mechanical approach applied to the modeling of structures and spectra of mixed molecular clusters $\operatorname{Ar}_{n}(\mathrm{HCl})_{m}$ and $\operatorname{Ar}_{n}(\mathrm{HF})_{m}$, J. Chem. Phys., 2004, 120, 3732-3743.

67 R. Wugt Larsen, P. Zielke and M. A. Suhm, Hydrogenbonded $\mathrm{OH}$ stretching modes of methanol clusters: A combined IR and Raman isotopomer study, J. Chem. Phys., 2007, 126, 194307.

68 M. A. Suhm, Hydrogen bond dynamics in alcohol clusters, Adv. Chem. Phys., 2009, 142, 1-57.

69 C. Emmeluth, V. Dyczmons, T. Kinzel, P. Botschwina, M. A. Suhm and M. Yáñez, Combined jet relaxation and quantum chemical study of the pairing preferences of ethanol, Phys. Chem. Chem. Phys., 2005, 7, 991-997.

70 D. Zimmermann, Th. Häber, H. Schaal and M. A. Suhm, Hydrogen bonded rings, chains and lassos: the case of t-butyl alcohol clusters, Mol. Phys., 2001, 99, 413-425.

71 M. Behrens, R. Fröchtenicht, M. Hartmann, J.-G. Siebers, U. Buck and F. C. Hagemeister, Vibrational spectroscopy of methanol and acetonitrile clusters in cold helium droplets, J. Chem. Phys., 1999, 111, 2436-2443.

72 J. B. Paul, C. P. Collier, R. J. Saykally, J. J. Scherer and A. O'Keefe, Direct measurement of water cluster concentrations by infrared cavity ringdown laser absorption spectroscopy, J. Phys. Chem. A, 1997, 101, 5211-5214.

73 M. Nedić, T. N. Wassermann, R. Wugt Larsen and M. A. Suhm, A combined Raman- and infrared jet study of mixed methanol-water and ethanol-water clusters, Phys. Chem. Chem. Phys., 2011, 13, 14050-14063.

74 M. Weimann, M. Fárník and M. A. Suhm, A first glimpse at the acidic proton vibrations in HCl-water clusters via supersonic jet FTIR spectroscopy, Phys. Chem. Chem. Phys., 2002, 4, 3933-3937.

75 K. R. Leopold, Hydrated acid clusters, Annu. Rev. Phys. Chem., 2011, 63, 327-349.

76 M. Miyazaki, A. Kawanishi, I. Nielsen, I. Alata, S.-i. Ishiuchi, C. Dedonder, C. Jouvet and M. Fujii, Ground state proton transfer in phenol- $\left(\mathrm{NH}_{3}\right)_{n}(n<11)$ clusters studied by mid-IR spectroscopy in 3-10 $\mu \mathrm{m}$ range, $J$. Phys. Chem. A, 2013, 117, 1522-1530.
77 M. Fárník, M. Weimann and M. A. Suhm, Acidic protons before take-off: a comparative jet Fourier transform infrared study of small $\mathrm{HCl}-$ and $\mathrm{HBr}-$ solvent complexes, J. Chem. Phys., 2003, 118, 10120-10136.

78 A. J. Huneycutt, R. J. Stickland, F. Hellberg and R. J. Saykally, Infrared cavity ringdown spectroscopy of acid-water clusters: $\mathrm{HCl}-\mathrm{H}_{2} \mathrm{O}, \mathrm{DCl}-\mathrm{D}_{2} \mathrm{O}$, and DCl- $\left(\mathrm{D}_{2} \mathrm{O}\right)_{2}$, J. Chem. Phys., 2003, 118, 1221-1229.

79 A. Gutberlet, G. Schwaab, Ö. Birer, M. Masia, A. Kaczmarek, H. Forbert, M. Havenith and D. Marx, Aggregation-induced dissociation of $\mathrm{HCl}\left(\mathrm{H}_{2} \mathrm{O}\right)_{4}$ below $1 \mathrm{~K}$, the smallest droplet of acid, Science, 2009, 324, 1545-1548.

80 S. D. Flynn, D. Skvortsov, A. M. Morrison, T. Liang, M. Y. Choi, G. E. Douberly and A. F. Vilesov, Infrared spectra of $\mathrm{HCl}-\mathrm{H}_{2} \mathrm{O}$ clusters in helium nanodroplets, J. Phys. Chem. Lett., 2010, 1, 52233-52238.

81 A. M. Morrison, S. D. Flynn, T. Liang and G. E. Douberly, Infrared spectroscopy of $(\mathrm{HCl})_{m}\left(\mathrm{H}_{2} \mathrm{O}\right)_{n}$ clusters in helium nanodroplets: definitive assignments in the $\mathrm{HCl}$ stretch region, J. Phys. Chem. A, 2010, 114, 8090-8098.

82 M. Weimann, M. Fárník, M. A. Suhm, M. E. Alikhani and J. Sadlej, Cooperative and anticooperative mixed trimers of $\mathrm{HCl}$ and methanol, J. Mol. Struct., 2006, 790, 18-26.

83 C. Emmeluth, M. A. Suhm and D. Luckhaus, A monomersin-dimers model for carboxylic acid dimers, J. Chem. Phys., 2003, 118, 2242-2255.

84 T. N. Wassermann, C. A. Rice, M. A. Suhm and D. Luckhaus, Hydrogen bonding lights up overtones in pyrazoles, J. Chem. Phys., 2007, 127, 234309.

85 Y. Matsumoto and K. Honma, NH stretching vibrations of pyrrole clusters studied by infrared cavity ringdown spectroscopy, J. Chem. Phys., 2007, 127, 184310.

86 I. Dauster, C. A. Rice, P. Zielke and M. A. Suhm, N-H $\cdots \pi$ interactions in pyrroles: systematic trends from the vibrational spectroscopy of clusters, Phys. Chem. Chem. Phys., 2008, 10, 2827-2835.

87 S. Hesse and M. A. Suhm, Conformation and aggregation of proline esters and their aromatic homologs: pyramidal vs. planar RR'N-H in hydrogen bonds, Z. Phys. Chem., 2009, 223, 579-604.

88 K. E. Otto, S. Hesse, T. N. Wassermann, C. A. Rice, M. A. Suhm, T. Stafforst and U. Diederichsen, Temperaturedependent intensity anomalies in amino acid esters: weak hydrogen bonds in protected glycine, alanine and valine, Phys. Chem. Chem. Phys., 2011, 13, 14119-14130.

89 J. J. Lee, M. Albrecht, C. A. Rice, M. A. Suhm, A. Stamm, M. Zimmer and M. Gerhards, Adaptive aggregation of peptide model systems, J. Phys. Chem. A, 2013, DOI: 10.1021/jp400056n.

90 S. Hesse, T. N. Wassermann and M. A. Suhm, Brightening and locking a weak and floppy $\mathrm{N}-\mathrm{H}$ chromophore: the case of pyrrolidine, J. Phys. Chem. A, 2010, 114, 10492-10499.

91 L. Du and H. G. Kjaergaard, Fourier transform infrared spectroscopy and theoretical study of dimethylamine dimer in the gas phase, J. Phys. Chem. A, 2011, 115, 12097-12104. 
92 C. Pfaffen, D. Infanger, P. Ottiger, H.-M. Frey and S. Leutwyler, $\mathrm{N}-\mathrm{H} \cdots \pi$ hydrogen-bonding and large-amplitude tipping vibrations in jet-cooled pyrrole-benzene, Phys. Chem. Chem. Phys., 2011, 13, 14110-14118.

93 A. L. Sobolewski, W. Domcke, C. Dedonder-Lardeux and C. Jouvet, Excited-state hydrogen detachment and hydrogen transfer driven by repulsive ${ }^{1} \pi \sigma^{*}$ states: a new paradigm for nonradiative decay in aromatic biomolecules, Phys. Chem. Chem. Phys., 2002, 4, 1093-1100.

94 M. Albrecht, A. Borba, K. Le Barbu-Debus, B. Dittrich, R. Fausto, S. Grimme, A. Mahjoub, M. Nedić, U. Schmitt, L. Schrader, M. A. Suhm, A. Zehnacker-Rentien and J. Zischang, Chirality influence on the aggregation of methyl mandelate, New J. Chem., 2010, 34, 1266-1285.

95 J. Altnöder, A. Bouchet, J. J. Lee, K. E. Otto, M. A. Suhm and A. Zehnacker-Rentien, Chirality-dependent balance between hydrogen bonding and London dispersion in isolated ( \pm )-1-indanol clusters, Phys. Chem. Chem. Phys., 2013, DOI: 10.1039/c3cp50708d.

96 Y. Matsumoto, J.-i. Iwamoto and K. Honma, Contribution of the $\pi$ electron to the $\mathrm{N}-\mathrm{H} \cdots \mathrm{O}=\mathrm{C}$ hydrogen bond: IR spectroscopic studies of the jet-cooled pyrrole-acetone binary clusters, Phys. Chem. Chem. Phys., 2012, 14, 12938.

97 W. H. James III, E. G. Buchanan, C. W. Müller, J. C. Dean, D. Kosenkov, L. V. Slipchenko, L. Guo, A. G. Reidenbach, S. H. Gellman and T. S. Zwier, Evolution of amide stacking in larger $\gamma$-peptides: triamide H-bonded cycles, J. Phys. Chem. A, 2011, 115, 13783-13798.

98 C. A. Rice, I. Dauster and M. A. Suhm, Infrared spectroscopy of pyrrole-2-carboxaldehyde and its dimer: a planar $\beta$-sheet peptide model?, J. Chem. Phys., 2007, 126, 134313.

99 M. Gerhards, C. Unterberg, A. Gerlach and A. Jansen, $\beta$-Sheet model systems in the gas phase: structures and vibrations of Ac-Phe-NHMe and its dimer (Ac-Phe-NHMe) ${ }_{2}$, Phys. Chem. Chem. Phys., 2004, 6, 2682-2690.

100 A. R. Al-Rabaa, E. Bréhéret, F. Lahmani and A. Zehnacker, Enantiodifferentiation in jet-cooled van der Waals complexes of chiral molecules, Chem. Phys. Lett., 1995, 237, 480-484.

101 N. Borho, T. Häber and M. A. Suhm, Chiral self-recognition in the gas phase: the case of glycidol dimers, Phys. Chem. Chem. Phys., 2001, 3, 1945-1948.

102 A. Zehnacker and M. A. Suhm, Chirality recognition between neutral molecules in the gas phase, Angew. Chem., Int. Ed., 2008, 47, 6970-6992.

103 K. Le Barbu, V. Brenner, Ph. Millié, F. Lahmani and A. Zehnacker-Rentien, An experimental and theoretical study of jet-cooled complexes of chiral molecules: the role of dispersive forces in chiral discrimination, J. Phys. Chem. A, 1998, 102, 128-137.

104 D. Scuderi, K. Le Barbu-Debus and A. Zehnacker, The role of weak hydrogen bonds in chiral recognition, Phys. Chem. Chem. Phys., 2011, 13, 17916-17929.

105 M. Albrecht, V. A. Soloshonok, L. Schrader, M. Yasumoto and M. A. Suhm, Chirality-dependent sublimation of $\alpha$-(trifluoromethyl)-lactic acid: relative vapor pressures of racemic, eutectic, and enantiomerically pure forms, and vibrational spectroscopy of isolated $(S, S)$ and $(S, R)$ dimers, J. Fluorine Chem., 2010, 131, 495-504.

106 R. Tonner, V. A. Soloshonok and P. Schwerdtfeger, Theoretical investigations into the enantiomeric and racemic forms of $\alpha$-(trifluoromethyl)lactic acid, Phys. Chem. Chem. Phys., 2011, 13, 811-817.

107 M. A. Suhm and M. Albrecht, Comment on "Theoretical investigations into the enantiomeric and racemic forms of $\alpha$-(trifluoromethyl)lactic acid" by R. Tonner, V. A. Soloshonok and P. Schwerdtfeger, Phys. Chem. Chem. Phys., 2011, 13, 811-817, Phys. Chem. Chem. Phys., 2011, 13, 4159-4160.

108 M. Albrecht, J. Will and M. A. Suhm, Chirality recognition in menthol and neomenthol: Preference for homoconfigurational aggregation, Angew. Chem., Int. Ed., 2010, 49, 6203-6206.

109 N. Borho and M. A. Suhm, Glycidol dimer: anatomy of a molecular handshake, Phys. Chem. Chem. Phys., 2002, 4, 2721-2732.

110 N. Borho and M. A. Suhm, Tailor-made aggregates of $\alpha$-hydroxy esters in supersonic jets, Phys. Chem. Chem. Phys., 2004, 6, 2885-2890.

111 T. B. Adler, N. Borho, M. Reiher and M. A. Suhm, Chiralityinduced switch in hydrogen-bond topology: tetrameric methyl lactate clusters in the gas phase, Angew. Chem., Int. Ed., 2006, 45, 3440-3445.

112 A. Sharma, I. Reva, R. Fausto, S. Hesse, Z. Xue, M. A. Suhm, S. K. Nayak, R. Sathishkumar, R. Pal and T. N. Guru Row, Conformation-changing aggregation in hydroxyacetone: a combined low-temperature FTIR, jet, and crystallographic study, J. Am. Chem. Soc., 2011, 133, 20194-20207.

113 J. Altnöder, J. J. Lee, K. E. Otto and M. A. Suhm, Molecular recognition in glycolaldehyde, the simplest sugar: two isolated hydrogen bonds win over one cooperative pair, ChemistryOpen, 2012, 1, 269-275.

114 N. Borho and M. A. Suhm, Self-organization of lactates in the gas phase, Org. Biomol. Chem., 2003, 1, 4351-4358.

115 H. Schaal, T. Häber and M. A. Suhm, Hydrogen bonding in 2-propanol: the effect of fluorination, J. Phys. Chem. A, 2000, 104, 265-274.

116 T. Scharge, C. Emmeluth, T. Häber and M. A. Suhm, Competing hydrogen bond topologies in 2-fluoroethanol dimer, J. Mol. Struct., 2006, 786, 86-95.

117 T. Scharge, D. Luckhaus and M. A. Suhm, Observation and quantification of the hydrogen bond effect on $\mathrm{O}-\mathrm{H}$ overtone intensities in an alcohol dimer, Chem. Phys., 2008, 346, 167-175.

118 M. Buck, Trifluoroethanol and colleagues: cosolvents come of age. Recent studies with peptides and proteins, Q. Rev. Biophys., 1998, 31, 297-355.

119 T. Scharge, C. Cézard, P. Zielke, A. Schütz, C. Emmeluth and M. A. Suhm, A peptide co-solvent under scrutiny: selfaggregation of 2,2,2-trifluoroethanol, Phys. Chem. Chem. Phys., 2007, 9, 4472-4490.

120 M. Hippler, S. Hesse and M. A. Suhm, Quantum-chemical study and FTIR jet spectroscopy of $\mathrm{CHCl}_{3}-\mathrm{NH}_{3}$ association 
in the gas phase, Phys. Chem. Chem. Phys., 2010, 12, 13555-13565.

121 B. Michielsen, J. J. J. Dom, B. J. van der Veken, S. Hesse, Z. Xue, M. A. Suhm and W. A. Herrebout, The complexes of halothane with benzene: the temperature dependent direction of the complexation shift of the aliphatic C-H stretching, Phys. Chem. Chem. Phys., 2010, 12, 14034-14044.

122 B. Michielsen, J. J. J. Dom, B. J. van der Veken, S. Hesse, M. A. Suhm and W. A. Herrebout, Solute-solvent interactions in cryosolutions: a study of halothane-ammonia complexes, Phys. Chem. Chem. Phys., 2012, 14, 6469-6478.

123 Z. Su, N. Borho and Y. Xu, Chiral self-recognition: direct spectroscopic detection of the homochiral and heterochiral dimers of propylene oxide in the gas phase, J. Am. Chem. Soc., 2006, 128, 17126-17131.

124 F. Kollipost, S. Hesse, J. J. Lee and M. A. Suhm, Dimers of cyclic carbonates: chirality recognition in battery solvents and energy storage, Phys. Chem. Chem. Phys., 2011, 13, 14176-14182.

125 C. Emmeluth and M. A. Suhm, A chemical approach towards the spectroscopy of carboxylic acid dimer isomerism, Phys. Chem. Chem. Phys., 2003, 5, 3094-3099.

126 S. Hesse and M. A. Suhm, On the low volatility of cyclic esters: an infrared spectroscopy comparison between dimers of $\gamma$-butyrolactone and methyl propionate, Phys. Chem. Chem. Phys., 2009, 11, 11157-11170.

127 M. K. Kunzmann, S. Bauerecker, M. A. Suhm and R. Signorell, Spectroscopic characterization of $\mathrm{N}_{2} \mathrm{O}$ aggregates: from clusters to the particulate state, Spectrochim. Acta, Part A, 2003, 59, 2855-2865.

128 R. Signorell, M. Jetzki, M. Kunzmann and R. Ueberschaer, Unraveling the origin of band shapes in infrared spectra of $\mathrm{N}_{2} \mathrm{O}-{ }^{12} \mathrm{CO}_{2}$ and ${ }^{12} \mathrm{CO}_{2}{ }^{-}{ }^{13} \mathrm{CO}_{2}$ ice particles, J. Phys. Chem. A, 2006, 110, 2890-2897.

129 Y. Liu, M. A. Suhm and P. Botschwina, Supersonic jet FTIR and quantum chemical investigations of ammonia/acetylene clusters, Phys. Chem. Chem. Phys., 2004, 6, 4642-4651.

130 E. J. Ocola, A. A. Al-Saadi, C. Mlynek, H. Hopf and J. Laane, Intramolecular $\pi$-type hydrogen bonding and conformations of 3-cyclopenten-1-ol. 2. Infrared and Raman spectral studies at high temperatures, J. Phys. Chem. A, 2010, 114, 7457-7461.

131 B. J. Miller, J. R. Lane and H. G. Kjaergaard, Intramolecular $\mathrm{OH} \cdots \pi$ interactions in alkenols and alkynols, Phys. Chem. Chem. Phys., 2011, 13, 14183-14193.

132 M. Ortlieb and M. Havenith, Proton transfer in $(\mathrm{HCOOH})_{2}$ : an IR high-resolution spectroscopic study of the antisymmetric C-O stretch, J. Phys. Chem. A, 2007, 111, 7355-7363.

133 U. Buck, Properties of neutral clusters from scattering experiments, J. Phys. Chem., 1988, 92, 1023-1031.

134 M. Ehbrecht and F. Huisken, Vibrational spectroscopy of ethanol molecules and complexes selectively prepared in the gas phase and adsorbed on large argon clusters, J. Phys. Chem. A, 1997, 101, 7768-7777.

135 H. Fricke, A. Gerlach and M. Gerhards, Structure of a betasheet model system in the gas phase: analysis of the fingerprint region up to $10 \mu \mathrm{m}$, Phys. Chem. Chem. Phys., 2006, 8, 1660-1662.

136 F. Ito, Jet-cooled infrared spectra of the formic acid dimer by cavity ring-down spectroscopy: observation of the $\mathrm{C}-\mathrm{O}$ stretching region and vibrational analysis of the Fermitriad system, Chem. Phys. Lett., 2007, 447, 202-207.

137 J. M. Bakker, L. M. Aleese, G. von Helden and G. Meijer, The infrared absorption spectrum of the gas phase neutral benzoic acid monomer and dimer, J. Chem. Phys., 2003, 119, 11180-11185.

138 T. N. Wassermann, M. A. Suhm, P. Roubin and S. Coussan, Isomerization around $\mathrm{C}-\mathrm{C}$ and $\mathrm{C}-\mathrm{O}$ bonds in 1-propanol: collisional relaxation in supersonic jets and selective IR photo-isomerization in cryogenic matrices, J. Mol. Struct., 2012, 1025, 20-32.

139 Y. Liu, M. Weimann and M. A. Suhm, Extension of panoramic cluster jet spectroscopy into the far infrared: low frequency modes of methanol and water clusters, Phys. Chem. Chem. Phys., 2004, 6, 3315-3319.

140 R. Wugt Larsen and M. A. Suhm, Cooperative organic hydrogen bonds: the librational modes of cyclic methanol clusters, J. Chem. Phys., 2006, 125, 154314.

141 R. Wugt Larsen and M. A. Suhm, The benefits of alternation and alkylation: large amplitude hydrogen bond librational modes of alcohol trimers and tetramers, Phys. Chem. Chem. Phys., 2010, 12, 8152-8157.

142 T. A. Blake, S. W. Sharpe and S. S. Xantheas, Rotationally resolved spectroscopy of a librational fundamental band of hydrogen fluoride, J. Chem. Phys., 2000, 113, 707-718.

143 M. Cirtog, P. Asselin, P. Soulard, B. Madebène and M. E. Alikhani, Intermolecular vibrations of $\left(\mathrm{CH}_{2}\right)_{2} \mathrm{O}-\mathrm{HF}$ and -DF hydrogen bonded complexes investigated by Fourier transform infrared spectroscopy and ab initio calculations, Phys. Chem. Chem. Phys., 2010, 12, 12299-12307.

144 J. Ceponkus, P. Uvdal and B. Nelander, Water tetramer, pentamer, and hexamer in inert matrices, J. Phys. Chem. A, 2012, 116, 4842-4850.

145 P. Zielke and M. A. Suhm, Raman jet spectroscopy of formic acid dimers: low frequency vibrational dynamics and beyond, Phys. Chem. Chem. Phys., 2007, 9, 4528-4534.

146 D. T. Anderson, S. Davis and D. J. Nesbitt, Probing hydrogen bond potentials via combination band spectroscopy: a near-IR study of the geared bend/van der Waals stretch intermolecular modes in $(\mathrm{HF})_{2}, J$. Chem. Phys., 1996, 104, 6225-6243.

147 F. N. Keutsch and R. J. Saykally, Water clusters: untangling the mysteries of the liquid, one molecule at a time, Proc. Natl. Acad. Sci. U. S. A., 2001, 98, 10533-10540.

148 M. Schütz, T. Bürgi, S. Leutwyler and T. Fischer, Intermolecular bonding and vibrations of 2-naphtol. $\mathrm{H}_{2} \mathrm{O}\left(\mathrm{D}_{2} \mathrm{O}\right)$, J. Chem. Phys., 1993, 99, 1469-1481.

149 K. Le Barbu-Debus, F. Lahmani, A. Zehnacker-Rentien, N. Guchhait, S. S. Panja and T. Chakraborty, Fluorescence spectroscopy of jet-cooled chiral (+/-)-indan-1-ol and its cluster with (+/-)-methyl- and ethyl-lactate, J. Chem. Phys., 2006, 125, 174305. 
150 C. P. Rodrigo, W. H. James III and T. S. Zwier, Singleconformation ultraviolet and infrared spectra of jet-cooled monolignols: p-coumaryl alcohol, coniferyl alcohol and sinapyl alcohol, J. Am. Chem. Soc., 2011, 133, 2632-2641.

151 I. N. Smirnova, A. Cuisset, F. Hindle, G. Mouret, R. Bocquet, O. Pirali and P. Roy, Gas-phase synchrotron FTIR spectroscopy of weakly volatile alkyl phosphonate and alkyl phosphate compounds: vibrational and conformational analysis in the terahertz/far-IR spectral domain, J. Phys. Chem. B, 2010, 114, 16936-16947.

152 A. S. Case, C. G. Heid, S. H. Kable and F. Fleming Crim, Dissociation energy and vibrational predissociation dynamics of the ammonia dimer, J. Chem. Phys., 2011, 135, 084312.

153 B. E. Rocher-Casterline, L. C. Ch'ng, A. K. Mollner and H. Reisler, Communication: determination of the bond dissociation energy $\left(D_{0}\right)$ of the water dimer, $\left(\mathrm{H}_{2} \mathrm{O}\right)_{2}$, by velocity map imaging, J. Chem. Phys., 2011, 134, 211101.

154 C. Wickleder, T. Droz, T. Bürgi and S. Leutwyler, Accurate intermolecular binding energies of 1-naphthol to benzene and cyclohexane, Chem. Phys. Lett., 1997, 264, 257-264.

155 S. Ebbinghaus, S. J. Kim, M. Heyden, X. Yu, U. Heugen, M. Gruebele, D. M. Leitner and M. Havenith, An extended dynamical hydration shell around proteins, Proc. Natl. Acad. Sci. U. S. A., 2007, 104, 20749-20752.

156 T. Ding, R. Li, J. A. Zeitler, T. L. Huber, L. F. Gladden, A. P. J. Middelberg and R. J. Falconer, Terahertz and far infrared spectroscopy of alanine-rich peptides having variable ellipticity, Opt. Express, 2010, 18, 27431-27444.

157 C. Sándorfy, Hydrogen bonding: How much anharmonicity?, J. Mol. Struct., 2006, 790, 50-54.

158 V. Cervetto, J. Helbing, J. Bredenbeck and P. Hamm, Double-resonance versus pulsed Fourier transform twodimensional infrared spectroscopy: an experimental and theoretical comparison, J. Chem. Phys., 2004, 121, 5935-5942.

159 M. Cho, Coherent two-dimensional optical spectroscopy, Chem. Rev., 2008, 108, 1331-1418.

160 M. Thomas, M. Brehm, R. Fligg, P. Vöhringer and B. Kirchner, Computing vibrational spectra from $a b$ initio molecular dynamics, Phys. Chem. Chem. Phys., 2013, 15, 6608-6622.

161 T. Omi, H. Shitomi, N. Sekiya, K. Takazawa and M. Fujii, Nonresonant ionization detected IR spectroscopy for the vibrational study in a supersonic jet, Chem. Phys. Lett., 1996, 252, 287-293.

162 H. G. Kjaergaard, D. L. Howard, D. P. Schofield, T. W. Robinson, S.-i. Ishiuchi and M. Fujii, OH- and $\mathrm{CH}$-stretching overtone spectra of catechol, J. Phys. Chem. A, 2002, 106, 258-266.

163 B. J. Miller, H. G. Kjaergaard, K. Hattori, S.-i. Ishiuchi and M. Fujii, The most stable conformer of benzyl alcohol, Chem. Phys. Lett., 2008, 466, 21-26.

164 F. Lahmani, M. Broquier and A. Zehnacker-Rentien, The $o$-cyanophenol dimer as studied by laser-induced fluorescence and IR fluorescence dip spectroscopy: a study of a symmetrical double hydrogen bond, Chem. Phys. Lett., 2002, 354, 337-348.

165 C. Lauzin, K. Didriche, J. Liévin, M. Herman and A. Perrin, Investigation of the $\mathrm{C}_{2} \mathrm{H}_{2}-\mathrm{CO}_{2}$ van der Waals complex in the overtone range using cw cavity ring-down spectroscopy, J. Chem. Phys., 2009, 130, 204306.

166 M. Hippler, C. Mohr, K. A. Keen and E. D. McNaghten, Cavity-enhanced resonant photoacoustic spectroscopy with optical feedback cw diode lasers: a novel technique for ultratrace gas analysis and high-resolution spectroscopy, J. Chem. Phys., 2010, 133, 044308.

167 M. Hippler and M. Quack, Overtone spectroscopy by vibrationally assisted dissociation and photofragment ionization, Chem. Phys. Lett., 1994, 231, 75-80.

168 C.-C. Chuang, S. N. Tsang, W. Klemperer and H.-C. Chang, Reassignment of the $11537 \mathrm{~cm}^{-1}$ band of hydrogen fluoride dimer and observation of the intermolecular combination mode $3 \nu_{1}+\nu_{4}$, J. Phys. Chem. A, 1997, 101, 6702-6708.

169 M. A. Suhm, J. T. Farrell, Jr., A. McIlroy and D. J. Nesbitt, High resolution $1.3 \mu \mathrm{m}$ overtone spectroscopy of $\mathrm{HF}$ dimer in a slit jet: $K_{\mathrm{a}}=0 \leftarrow 0$ and $K_{\mathrm{a}}=1 \leftarrow 0$ subbands of $v_{\text {acc }}=2 \leftarrow 0, J$. Chem. Phys., 1992, 97, 5341-5354.

170 K. Liu, M. Dulligan, I. Bezel, A. Kolessov and C. Wittig, Quenching of interconversion tunneling: the free $\mathrm{HCl}$ stretch first overtone of $(\mathrm{HCl})_{2}, J$. Chem. Phys., 1998, 108, 9614-9616.

171 S. A. Nizkorodov, M. Ziemkiewicz, D. J. Nesbitt and A. E. W. Knight, Overtone spectroscopy of $\mathrm{H}_{2} \mathrm{O}$ clusters in the $v_{\mathrm{OH}}=2$ manifold: infrared-ultraviolet vibrationally mediated dissociation studies, J. Chem. Phys., 2005, 122, 194316.

172 Y. He, H. B. Müller, M. Quack and M. A. Suhm, High resolution FTIR and diode laser supersonic jet spectroscopy of the $N=2 \mathrm{HF}$ stretching polyad in $(\mathrm{HF})_{2}$ and (HFDF): hydrogen bond switching and predissociation dynamics, Z. Phys. Chem., 2007, 221, 1581-1645.

173 M. Hippler, L. Oeltjen and M. Quack, High-resolution continuous-wave-diode laser cavity ring-down spectroscopy of the hydrogen fluoride dimer in a pulsed slit jet expansion: two components of the $N=2$ triad near $1.3 \mu \mathrm{m}$, J. Phys. Chem. A, 2007, 111, 12659-12668.

174 K. Didriche, C. Lauzin, T. Foldes, D. Golebiowski, M. Herman and C. Leforestier, High resolution overtone spectroscopy of the acetylene van der Waals dimer, $\left({ }^{12} \mathrm{C}_{2} \mathrm{H}_{2}\right)_{2}$, Phys. Chem. Chem. Phys., 2011, 13, 14010-14018.

175 M. A. Suhm, How broad are water dimer bands?, Science, 2004, 304, 823.

176 M. Fárník, C. Steinbach, M. Weimann, U. Buck, N. Borho and M. A. Suhm, Size-selective vibrational spectroscopy of methyl glycolate clusters: comparison with ragout-jet FTIR spectroscopy, Phys. Chem. Chem. Phys., 2004, 6, 4614-4620.

177 M. Fárník, M. Weimann, C. Steinbach, U. Buck, N. Borho, T. B. Adler and M. A. Suhm, Size-selected methyl lactate clusters: fragmentation and spectroscopic fingerprints of chiral recognition, Phys. Chem. Chem. Phys., 2006, 8, 1148-1158. 
178 F. Huisken, M. Kaloudis, A. Kulcke, C. Laush and J. M. Lisy, Vibrational spectroscopy of small $(\mathrm{HF})_{n}$ clusters ( $n=4-8)$ in size-selected molecular beams, J. Chem. Phys., 1995, 103, 5366-5377.

179 G. E. Douberly and R. E. Miller, The growth of HF polymers in helium nanodroplets: probing the barriers to ring insertion, J. Phys. Chem. B, 2003, 107, 4500-4507.

180 F. O. Talbot and J. P. Simons, Infrared ion dip and ultraviolet spectroscopy of 4-phenyl imidazole, its tautomer, 5-phenyl imidazole, and its multiply hydrated clusters, Eur. Phys. J. D, 2002, 20, 389-398.

181 H. Fricke, K. Schwing, A. Gerlach, C. Unterberg and M. Gerhards, Investigations of the water clusters of the protected amino acid Ac-Phe-OMe by applying IR/UV double resonance spectroscopy: microsolvation of the backbone, Phys. Chem. Chem. Phys., 2010, 12, 3511-3521.

182 R. Kusaka, Y. Inokuchi and T. Ebata, Vibrational energy relaxation of benzene dimer and trimer in the $\mathrm{CH}$ stretching region studied by picosecond time-resolved IR-UV pumpprobe spectroscopy, J. Chem. Phys., 2012, 136, 044304.

183 J. C. Dean, E. G. Buchanan, W. H. James III, A. Gutberlet, B. Biswas, P. V. Ramachandran and T. S. Zwier, Conformation-specific spectroscopy and populations of diastereomers of a model monolignol derivative: chiral effects in a triol chain, J. Phys. Chem. A, 2011, 115, 8464-8478.

184 T. S. Zwier, The spectroscopy of solvation in hydrogenbonded aromatic clusters, Annu. Rev. Phys. Chem., 1996, 47, 205-241.

185 T. Hamashima, K. Mizuse and A. Fujii, Spectral signatures of four-coordinated sites in water clusters: infrared spectroscopy of phenol- $\left(\mathrm{H}_{2} \mathrm{O}\right)_{n}(\sim 20<n<\sim 50)$, J. Phys. Chem. A, 2011, 115, 620-625.

186 C. C. Pradzynski, R. M. Forck, T. Zeuch, P. Slavicek and U. Buck, A fully size-resolved perspective on the crystallization of water clusters, Science, 2012, 337, 1529-1532.

187 Y. Matsuda, K. Ohta, N. Mikami and A. Fujii, Infrared spectroscopy for acetone and its dimer based on photoionization detection with tunable coherent vacuumultraviolet light, Chem. Phys. Lett., 2007, 442, 217-219.

188 R. M. Forck, C. C. Pradzynski, S. Wolff, M. Ončák, P. Slavíček and T. Zeuch, Size resolved infrared spectroscopy of $\mathrm{Na}\left(\mathrm{CH}_{3} \mathrm{OH}\right)_{n}(n=4-7)$ clusters in the $\mathrm{OH}$ stretching region: unravelling the interaction of methanol clusters with a sodium atom and the emergence of the solvated electron, Phys. Chem. Chem. Phys., 2012, 14, 3004-3016.

189 R. M. Forck, J. M. Dieterich, C. C. Pradzynski, A. L. Huchting, R. A. Mata and T. Zeuch, Structural diversity in sodium doped water trimers, Phys. Chem. Chem. Phys., 2012, 14, 9054-9057.

190 H. B. Fu, Y. J. Hu and E. R. Bernstein, IR + vacuum ultraviolet (118 nm) nonresonant ionization spectroscopy of methanol monomers and clusters: neutral cluster distribution and size-specific detection of the $\mathrm{OH}$ stretch vibrations, J. Chem. Phys., 2006, 124, 024302.

191 Y. Xu, J. Van Wijngaarden and W. Jager, Microwave spectroscopy of ternary and quaternary van der Waals clusters, Int. Rev. Phys. Chem., 2005, 24, 301-338.
192 Z. Kisiel, A. Lesarri, J. L. Neill, M. T. Muckle and B. H. Pate, Structure and properties of the $(\mathrm{HCl})_{2}\left(\mathrm{H}_{2} \mathrm{O}\right)$ cluster observed by chirped-pulse Fourier transform microwave spectroscopy, Phys. Chem. Chem. Phys., 2011, 13, 13912-13919.

193 E. J. Cocinero, A. Lesarri, P. Écija, F. J. Basterretxea, J.-U. Grabow, J. A. Fernández and F. Castaño, Ribose found in the gas phase, Angew. Chem., Int. Ed., 2012, 51, 3119-3124.

194 V. V. Matylitsky, C. Riehn, M. F. Gelin and B. Brutschy, The formic acid dimer $(\mathrm{HCOOH})_{2}$ probed by time-resolved structure selective spectroscopy, J. Chem. Phys., 2003, 119, 10553-10562.

195 M. C. D. Tayler, B. Ouynag and B. J. Howard, Unraveling the spectroscopy of coupled intramolecular tunneling modes: a study of double proton transfer in the formicacetic acid complex, J. Chem. Phys., 2011, 134, 054316.

196 G. G. Brown, B. C. Dian, K. O. Douglass, S. M. Geyer, S. T. Shipman and B. H. Pate, A broadband Fourier transform microwave spectrometer based on chirped pulse excitation, Rev. Sci. Instrum., 2008, 79, 053103.

197 P. Zielke and M. A. Suhm, Concerted proton motion in hydrogen-bonded trimers: a spontaneous Raman scattering perspective, Phys. Chem. Chem. Phys., 2006, 8, 2826-2830.

198 P. Ottiger, S. Leutwyler and H. Köppel, Vibrational quenching of excitonic splittings in H-bonded molecular dimers: the electronic Davydov splittings cannot match experiment, J. Chem. Phys., 2012, 136, 174308.

199 N. O. B. Lüttschwager, T. N. Wassermann, S. Coussan and M. A. Suhm, Periodic bond breaking and making in the electronic ground state on a sub-picosecond timescale: $\mathrm{OH}$ bending spectroscopy of malonaldehyde in the frequency domain at low temperature, Phys. Chem. Chem. Phys., 2010, 12, 8201-8207.

200 T. N. Wassermann, P. Zielke, J. J. Lee, C. Cézard and M. A. Suhm, Structural preferences, argon nanocoating, and dimerization of $n$-alkanols as revealed by $\mathrm{OH}$ stretching spectroscopy in supersonic jets, J. Phys. Chem. A, 2007, 111, 7437-7448.

201 A. Borba, M. Albrecht, A. Gómez-Zavaglia, L. Lapinski, M. J. Novak, M. A. Suhm and R. Fausto, Dimer formation in nicotinamide and picolinamide in the gas and condensed phases probed by infrared spectroscopy, Phys. Chem. Chem. Phys., 2008, 10, 7010-7021.

202 C. Callegari, K. K. Lehmann, R. Schmied and G. Scoles, Helium nanodroplet isolation rovibrational spectroscopy: methods and recent results, J. Chem. Phys., 2001, 115, 10090-10110.

203 T. N. Wassermann, D. Luckhaus, S. Coussan and M. A. Suhm, Proton tunneling estimates for malonaldehyde vibrations from supersonic jet and matrix quenching experiments, Phys. Chem. Chem. Phys., 2006, 8, 2344-2348.

204 N. O. B. Lüttschwager, T. N. Wassermann, S. Coussan and M. A. Suhm, Vibrational tuning of the hydrogen transfer in malonaldehyde - a combined FTIR and Raman jet study, Mol. Phys., 2013, DOI: 10.1080/00268976.2013.798042. 
205 A. Gómez-Zavaglia and R. Fausto, Self-Aggregation in Pyrrole: Matrix Isolation, Solid State Infrared Spectroscopy, and DFT Study, J. Phys. Chem. A, 2004, 108, 6953-6967.

206 J. J. Lee, S. Höfener, W. Klopper, T. N. Wassermann and M. A. Suhm, Origin of the argon nanocoating shift in the $\mathrm{OH}$ stretching fundamental of $n$-propanol: a combined experimental and quantum chemical study, J. Phys. Chem. C, 2009, 113, 10929-10938.

207 A. McIlroy, R. Lascola, C. M. Lovejoy and D. J. Nesbitt, Structural dependence of HF vibrational red shifts in $\mathrm{Ar}_{n} \mathrm{HF}, n=1-4$, via high-resolution slit jet infrared spectroscopy, J. Phys. Chem., 1991, 95, 2636-2644.

208 I. Kalkman, C. Brand, T.-B. C. Vu, W. L. Meerts, Y. N. Svartsov, O. Dopfer, X. Tong, K. Müller-Dethlefs, S. Grimme and M. Schmitt, The structure of phenol- $\mathrm{Ar}_{n}$ $(n=1,2)$ clusters in their $S_{0}$ and $S_{1}$ states, J. Chem. Phys., 2009, 130, 224303.

209 J. P. Perchard and Z. Mielke, Anharmonicity and hydrogen bonding I. A near-infrared study of methanol trapped in nitrogen and argon matrices, Chem. Phys., 2001, 264, 221-234.

210 E. Knözinger, P. Beichert, J. Hermeling and O. Schrems, Matrix isolation of acetonitrile clusters from a pulsed supersonic beam, J. Phys. Chem., 1993, 97, 1324-1331.

211 M. Gantenberg, M. Halupka and W. Sander, Dimerization of formic acid - an example of a noncovalent reaction mechanism, Chem.-Eur. J., 2000, 6, 1865-1869.

$212 \mathrm{H}$. Frei and G. C. Pimentel, Infrared induced photochemical processes in matrices, Annu. Rev. Phys. Chem., 1985, 36, 491-524.

213 A. Sharma, I. Reva and R. Fausto, Conformational switching induced by near-infrared laser irradiation, J. Am. Chem. Soc., 2009, 131, 8752-8753.

214 K. Marushkevich, L. Khriachtchev, J. Lundell, A. Domanskaya and M. Räsänen, Matrix isolation and ab initio study of trans-trans and trans-cis dimers of formic acid, J. Phys. Chem. A, 2010, 114, 3495-3502.

215 J. Ceponkus, P. Uvdal and B. Nelander, On the structure of the matrix isolated water trimer, J. Chem. Phys., 2011, 134, 064309.

216 M. Y. Choi, G. E. Douberly, T. M. Falconer, W. K. Lewis, C. M. Lindsay, J. M. Merritt, P. L. Stiles and R. E. Miller, Infrared spectroscopy of helium nanodroplets: novel methods for physics and chemistry, Int. Rev. Phys. Chem., 2006, 25, 15-75.
217 J. P. Toennies and A. F. Vilesov, Superfluid helium droplets: a uniquely cold nanomatrix for molecules and molecular complexes, Angew. Chem., Int. Ed., 2004, 43, 2622-2648.

218 M. N. Slipchenko, S. Kuma, T. Momose and A. F. Vilesov, Intense pulsed helium droplet beams, Rev. Sci. Instrum., 2002, 73, 3600-3605.

219 F. Madeja, M. Havenith, K. Nauta, R. E. Miller, J. Chocholousova and P. Hobza, Polar isomer of formic acid dimers formed in helium droplets, J. Chem. Phys., 2004, 120, 10554-10560.

220 M. N. Slipchenko, K. E. Kuyanov, B. G. Sartakov and A. F. Vilesov, Infrared intensity in small ammonia and water clusters, J. Chem. Phys., 2006, 124, 241101.

221 M. Nedić and M. A. Suhm, Detailed assignment of the $\mathrm{CH}$ chromophores in methyl mandelate and mandelic acid: a multi-experimental approach using polarized FTIR microspectroscopy of sublimated crystals, Cryst. Growth Des., 2012, 12, 1933-1942.

222 A. Ramos, B. Maté, G. Tejeda, J. M. Fernández and S. Montero, Raman spectroscopy of hypersonic shock waves, Phys. Rev. E, 2000, 62, 4940-4945.

223 R. S. Ruoff, T. D. Klots, T. Emilsson and H. S. Gutowsky, Relaxation of conformers and isomers in seeded supersonic jets of inert gases, J. Chem. Phys., 1990, 93, 3142-3150.

224 P. D. Godfrey, R. D. Brown and F. M. Rodgers, The missing conformers of glycine and alanine: relaxation in seeded supersonic jets, J. Mol. Struct., 1996, 376, 65-81.

225 U. Erlekam, M. Frankowski, G. von Helden and G. Meijer, Cold collisions catalyse conformational conversion, Phys. Chem. Chem. Phys., 2007, 9, 3786-3789.

226 V. Mozhayskiy, M. N. Slipchenko, V. K. Adamchuk and A. F. Vilesov, Use of helium nanodroplets for assembly, transport and surface deposition of large molecular and atomic clusters, J. Chem. Phys., 2007, 127, 094701.

227 X. de Ghellinck d'Elseghem Vaernewijck, K. Didriche, C. Lauzin, A. Rizopoulos, M. Herman and S. Kassi, Cavity enhanced FTIR spectroscopy using a femto OPO absorption source, Mol. Phys., 2011, 109, 2173-2179.

228 M. Quack, J. Stohner and M. Willeke, High-resolution spectroscopic studies and theory of parity violation in chiral molecules, Annu. Rev. Phys. Chem., 2008, 59, 741-769. 